NASA/TM-2009-215704

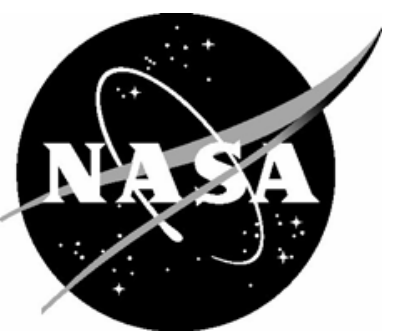

\title{
A Computational Approach for Probabilistic Analysis of Water Impact Simulations
}

Lucas G. Horta, Brian H. Mason, and Karen H. Lyle

Langley Research Center, Hampton, Virginia 


\section{NASA STI Program ... in Profile}

Since its founding, NASA has been dedicated to the advancement of aeronautics and space science. The NASA scientific and technical information (STI) program plays a key part in helping NASA maintain this important role.

The NASA STI program operates under the auspices of the Agency Chief Information Officer. It collects, organizes, provides for archiving, and disseminates NASA's STI. The NASA STI program provides access to the NASA Aeronautics and Space Database and its public interface, the NASA Technical Report Server, thus providing one of the largest collections of aeronautical and space science STI in the world. Results are published in both non-NASA channels and by NASA in the NASA STI Report Series, which includes the following report types:

- TECHNICAL PUBLICATION. Reports of completed research or a major significant phase of research that present the results of NASA programs and include extensive data or theoretical analysis. Includes compilations of significant scientific and technical data and information deemed to be of continuing reference value. NASA counterpart of peerreviewed formal professional papers, but having less stringent limitations on manuscript length and extent of graphic presentations.

- TECHNICAL MEMORANDUM. Scientific and technical findings that are preliminary or of specialized interest, e.g., quick release reports, working papers, and bibliographies that contain minimal annotation. Does not contain extensive analysis.

- CONTRACTOR REPORT. Scientific and technical findings by NASA-sponsored contractors and grantees.
- CONFERENCE PUBLICATION. Collected papers from scientific and technical conferences, symposia, seminars, or other meetings sponsored or co-sponsored by NASA.

- SPECIAL PUBLICATION. Scientific, technical, or historical information from NASA programs, projects, and missions, often concerned with subjects having substantial public interest.

- TECHNICAL TRANSLATION. Englishlanguage translations of foreign scientific and technical material pertinent to NASA's mission.

Specialized services also include creating custom thesauri, building customized databases, and organizing and publishing research results.

For more information about the NASA STI program, see the following:

- Access the NASA STI program home page at http://www.sti.nasa.gov

- E-mail your question via the Internet to help@sti.nasa.gov

- Fax your question to the NASA STI Help Desk at $443-757-5803$

- Phone the NASA STI Help Desk at 443-757-5802

- Write to:

NASA STI Help Desk NASA Center for AeroSpace Information 7115 Standard Drive Hanover, MD 21076-1320 
NASA/TM-2009-215704

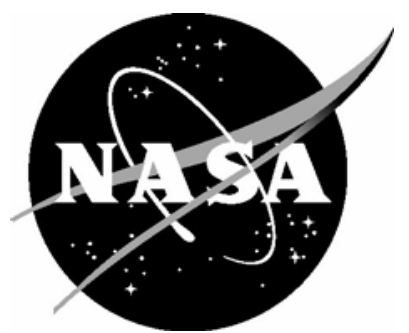

\section{A Computational Approach for Probabilistic Analysis of Water Impact Simulations}

Lucas G. Horta, Brian H. Mason, and Karen H. Lyle

Langley Research Center, Hampton, Virginia

National Aeronautics and

Space Administration 
Available from:

NASA Center for AeroSpace Information 7115 Standard Drive Hanover, MD 21076-1320 443-757-5802 


\title{
A Computational Approach for Probabilistic Analysis of Water Impact Simulations
}

\author{
Lucas G. Horta, Brian H. Mason, and Karen H. Lyle
}

\begin{abstract}
NASA's development of new concepts for the Crew Exploration Vehicle Orion presents many similar challenges to those worked in the sixties during the Apollo program. However, with improved modeling capabilities, new challenges arise. For example, the use of the commercial code LS-DYNA ${ }^{1}$, although widely used and accepted in the technical community, often involves high-dimensional, time consuming, and computationally intensive simulations. Because of the computational cost, these tools are often used to evaluate specific conditions and rarely used for statistical analysis. The challenge is to capture what is learned from a limited number of LS-DYNA simulations to develop models that allow users to conduct interpolation of solutions at a fraction of the computational time. For this problem, response surface models are used to predict the system time responses to a water landing as a function of capsule speed, direction, attitude, water speed, and water direction. Furthermore, these models can also be used to ascertain the adequacy of the design in terms of probability measures.

This paper presents a description of the LS-DYNA model, a brief summary of the response surface techniques, the analysis of variance approach used in the sensitivity studies, equations used to estimate impact parameters, results showing conditions that might cause injuries, and concluding remarks.
\end{abstract}

\section{Introduction}

During the development of the Apollo command module (CM), many studies were conducted to understand the behavior of the CM upon returning to earth. Because of the limited computational capabilities at the time, engineers made extensive use of experimental

\footnotetext{
${ }^{1}$ Trade names and trademarks are used in this report for identification only. Their usage does not constitute an official endorsement, either expressed or implied, by the National Aeronautics and Space Administration
} 
data to complement the relatively simple analyses. These studies were performed to understand the landing behavior under a variety of conditions. Interestingly, Apollo was initially to include a land-landing architecture. Thus, many of the early studies focused on assessing attenuation systems for landing on soil, sand, and clay surfaces [1-3]. However, the land-landing capability was abandoned for water splashdown, similar to the Mercury and Gemini capsules [4-5]. The exceptions to the water landing studies, were assessments of the capsule response in the event of a pad abort [6].

The Apollo water landing studies built on the extensive experience of the Mercury and Gemini programs. A wide range of experimental parameter studies, using subscale test articles, was conducted to evaluate the Apollo capsule performance. A primary interest of these studies was to understand the effect of landing attitude and longitudinal or horizontal velocity, as well as heatshield flexibility, on capsule response [7-11]. In addition, numerous full-scale tests were conducted to assess the capsule performance on more flight-like vehicles, e.g., References [12-14]. Finally, based on knowledge of probable landing conditions, a design envelope was established [15]. The purpose of the present work is to provide a probabilistic framework for the Orion Crew Module water landing performance boundary evaluation.

Results from two independent studies are reported here, using data from two sets of LSDYNA simulations, to illustrate the computational approach. One of the simulations used 4 parameters, whereas the second used 7 parameters to study landing of a rigid capsule on water. Although both efforts used the same LS-DYNA model, because the initial conditions used in each study are not identical, results cannot be easily combined into a single database. Instead results, in the form of time histories, from each study are treated separately and used to predict the Brinkley index [16] and to conduct statistical analysis. Fundamental differences in the approaches will be pointed out as results are presented.

For readers not familiar with the Brinkley index, it is a criterion, initially developed for emergency escape systems, to assess the probability of injury to a crew member when exposed to sudden acceleration pulses, like those observed during landings. Using local accelerations at the crew locations in all three directions, the criterion divides the risk of injury into three categories: 1) low risk corresponds to a $0.5 \%$ probability of injury, 2) moderate risk corresponds to a $5 \%$ probability of injury and, 3) high risk corresponds to a 
50\% probability of injury. Although there have been efforts to modify the thresholds set initially by Brinkley, the calculation of the index is unchanged and is what is used throughout the paper.

The paper presents a computational approach to assess impact conditions (velocities and orientation of capsule and impact plane) that could result in injuries to the crew when landing a rigid capsule on water. For this problem, a rigid LS-DYNA model is used and described first, followed by a detailed discussion of the formulation, the process to create the LS-DYNA database, sampling of the parameter space, sensitivity using conventional and analysis of variance, development of response surface models, and predictions of injury boundaries using the Brinkley index [16].

\section{Model Description}

The finite element (FE) model of Orion consists of three LS-DYNA [17] parts: the Orion capsule, a volume of air, and a volume of water. The capsule is built from Lagrangian rigid shell elements using 10307 elements, with an average element size of 3 in. Six nodes in the capsule model representing astronaut locations are used for extraction of acceleration results. For the water, a total of $1,282,500$ cubic elements ( 4 in. on a side) are used whereas the air uses 967,500 elements. Mass and mass moment of inertia properties for the capsule are given in Table 1. The volumes of air and water are composed of Eulerian eight-node brick elements with dimensions given in Fig. 1. The air is defined with a vacuum material, and the water is defined with a null material with a density of $9.3365 \mathrm{e}-5 \mathrm{lb}_{\mathrm{f}} \mathrm{s}^{2} / \mathrm{in}^{4}$ and a viscosity of $1.63 \mathrm{e}-7$.

The FE model is analyzed using the Arbitrary Lagrangian-Eulerian (ALE) formulation in LS-DYNA. The element time step for this model is $22.8 \mu \mathrm{s}$, resulting in an analysis time of 20 hrs per $100 \mathrm{~ms}$ of simulation time on a single node of a LINUX-based computer. Acceleration results are recorded to the nodout text file.

Because the impact conditions for the Orion are uncertain, seven critical impact parameters have been identified for study, as defined in Table 2, and are assumed to have a uniform distribution between the bounds provided in the table. Four of these parameters (horizontal velocity, vertical velocity, capsule pitch, and wave slope) are illustrated in Fig. 2. Also noted on the capsule are approximate crew locations 1-6. Transformations for both the capsule and the Euler grid (water and air) are defined in a LS-DYNA keyword file that is separate from 
the rest of the model. Separate keyword files for the Euler grid and the capsule are rotated, translated, and linked to the analysis run-stream using the *INCLUDE_TRANSFORM option.

\subsection{Mesh Convergence Study}

In this section, the effects of mesh refinement on the FE model are investigated. FE models with a rigid capsule at a 38-degree pitch angle and a vertical velocity of $50 \mathrm{ft} / \mathrm{s}$ are analyzed using 4 different Eulerian models. In all 4 cases, only the thickness of the brick elements in the vertical (global X) direction are varied; the in-plane dimensions for the brick elements are fixed at 4 in. wide by 4 in. long. Dimensions for the 4 cases are given in Table 3 . Also in Table 3 are the number of "interface" elements between air and water which are within 8 in. of the boundary surface; "far-field” elements are further away from the air/water boundary. Results of the mesh convergence study are shown in Fig 3 in terms of time histories of the Brinkley index for cases 1 to 4 . Time histories for case 2 are within $0.01 \%$ of case 1 (the baseline case), but the run time for case 2 is 30\% less than for case 1. The maximum Brinkley index for cases 3 and 4 are $4.0 \%$ and $1.6 \%$ higher than the baseline case, respectively. Unfortunately, the analysis time for cases 3 and 4 are 4.25 times longer than case 1 . Because of the computational expense of the more refined analyses (cases 3 and 4), the $4 \%$ difference in the refined and baseline analyses is considered small, and the solution is considered to be converged for case 1. Hence, results to follow will use the parameters in Case 1.

\section{Response Surface Description}

Response surface techniques provide a mathematical framework to synthesize models of systems for which users have only input/output data. For our purposes, these techniques will be used to provide time history predictions of water landings given a limited number of LSDYNA simulations. It is assumed that in order to solve the problem at hand, the amount of time to complete all required LS-DYNA runs is prohibited. Therefore an alternate way to obtain solutions, i.e., response surface models, is needed. Two techniques have been implemented and used; the first one was developed by Sacks [18] is often referred to as Kriging and the second technique is the Extended Radial Basis Functions [19-20]. Both 
techniques are briefly discussed to aid the reader in understanding some of the parameter settings used in our study.

\subsection{Kriging Response Surface}

In this approach, estimates of the system response are computed using the following expression

$$
\hat{y}(v)=\hat{\beta}+r(v)^{T} R^{-1}(Y-\hat{\beta} 1)
$$

with

$$
\begin{aligned}
& \hat{\beta}=\left(1^{T} R^{-1} 1\right)^{-1} 1^{T} R^{-1} Y \\
& R\left(v_{i}, v_{j}\right)=\exp \left[-\theta \sum_{k=1}^{m}\left(v_{i}^{k}-v_{j}^{k}\right)^{2}\right] \\
& r^{T}(v)=\left[\begin{array}{llll}
R\left(v, v_{1}\right) & R\left(v, v_{2}\right) & \ldots & R\left(v, v_{N}\right)
\end{array}\right] \\
& Y^{T}=\left[\begin{array}{llll}
y_{1} & y_{2} & \ldots & y_{N}
\end{array}\right] \\
& i=1,2, \ldots, N \text { and } j=1,2, \ldots, N
\end{aligned}
$$

where $v_{i}^{k}$ is $k^{\text {th }}$ component of the $i^{\text {th }}$ parameter vector, $v \in \mathbb{R}^{m \times 1}$ is a vector representing the parameters, $N$ is the population size, $y$ and $\hat{\beta}$ are scalars, $R \in \mathbb{R}^{N \times N}$ is a correlation matrix for the parameters, $r(v) \in \mathbb{R}^{N \times 1}$ is a correlation vector, $Y \in \mathbb{R}^{N \times 1}$ is a vector with $N$ response values, $\quad 1 \in \mathbb{R}^{N \times 1}$ is a vector whose elements are all ones, $\theta$ is a scalar parameter yet to be computed, and $m$ is the number of parameters. Although Eq. (3.1) is written for a system with a scalar output, the formulation is applied sequentially for cases with multiple outputs. A subtle but critical aspect of this formulation is the fact that the unknown parameter $\theta$, used to define the correlation matrix $R$, must be computed using maximum likelihood estimation. That is, $\theta$ is the value that maximizes the scalar metric

$$
L(\theta)=-\frac{1}{2}\left(N \ln \left(\sigma^{2}\right)+\ln |R|\right), \quad \theta \in[0, \infty]
$$

where the standard deviation is computed as

$$
\sigma^{2}=\frac{1}{N}(Y-\hat{\beta} 1)^{T} R^{-1}(Y-\hat{\beta} 1)
$$

Solving this scalar optimization problem produces a response surface fitting error with a Gaussian distribution that matches the $N$ response values. However, estimation of the parameter $\theta$ requires one to solve a scalar optimization for each output vector; which is 
conducted here using the genetic optimization algorithm in [21]. For problems containing $q$ outputs sampled $N$ times, to generate a Kriging surface requires $q N$ optimizations of the metric in Eq.(3.2). Although computationally intensive, the solutions are very accurate.

\subsection{Extended Radial Basis Functions}

The Extended Radial Basis Function (ERBF) method developed by Mullur and Messac in [19], approximates the system response as follows

$$
\hat{y}(v)=\sum_{i=1}^{N} \tau_{i} \psi\left(\left\|v-v_{i}\right\|\right)+\sum_{i=1}^{N} \phi_{i}\left(v-v_{i}\right)
$$

where $\psi$ is a radial basis function, $\tau_{i}$ is a scalar parameter solved as part of the least-square fitting process, and the function $\phi$ (known as the extended function) takes on different values depending upon the value of $v-v_{i}$, see [19] and [20]. To solve for $\phi$ requires solving a least square problem with $3 m N$ parameters. Since there are $N \tau_{i}$ values, the total number of parameters needed to compute an ERBF model is $N(3 m+1)$. In addition, to completely define $\phi$, two free parameters must be chosen: 1 ) the order of a local polynomial (set to 4 in this example), and 2) the smoothness parameter $\gamma$ (set to 0.15). Finally, the radial basis function $\psi$ is chosen as an exponentially decaying function $e^{-\left(\nu-v_{i}\right)^{2} / 2 r_{c}^{2}}$ with characteristic radius $r_{c}$ set to 0.15 .

In both Kriging and ERBF, scaling of the parameter variables $v$ is extremely important. In our implementation the absolute value of the maximum parameter value is used for scaling such that when variables are at their maximum value the normalized variable equals 1 .

\section{Parameter Sampling}

Parameter sampling is perhaps one of the most important steps when creating response surfaces. Techniques such as Latin Hypercube [22], random sampling, Hammersly [23], Doptimal[24], Halton-leap [25-26], and even manual setting of parameter variables are just a few of the possible means to prescribe a sample parameter. In our study D-optimal, Haltonleap, and manual parameter selection have been used at different times for different reasons. For example, in the beginning of the study the D-Optimal sampling approach was used because it would be followed by an analysis of variance study and this technique is ideally 
suited for that purpose. On the other hand, for the single variable sensitivity study, being conducted in parallel, manual sampling (heuristic approach) made the most sense. Nonetheless, for good coverage of the parameter range, Halton-leap deterministic sampling approach is also used.

Later in the paper, results for two independent studies using 4 and 7 independent variables are presented. For the 4-variable study, sampling of the parameter variables is conducted manually to study specific areas of concern and to facilitate sensitivity studies one parameter at a time. In contrast, the 7-parameter study used D-Optimal, Halton-leap, and manual sampling.

\section{Analysis of Variance}

Parameter sensitivity in most engineering fields in often associated with derivative calculations at specific points in the parameter space. However, for analysis of nonlinear systems under uncertainty, sensitivity studies are often conducted using Analysis of Variance (ANOVA). In classical ANOVA studies, data is collected from multiple experiments while varying all parameters (factors) and also varying one parameter at a time. These results are then used to assess the output response variance due to variations of a particular parameter as compared to the total output variance when varying all the parameters simultaneously. The ratio of these two variance contributions is a direct measure of the parameter importance.

Sobol in [27] and others [27-31] have studied the problem of global sensitivity analysis using variance measures. Sobol developed the "so called" Sobol indices to provide a measure of parameter sensitivity. In his formulation the variance is computed using the following expressions;

$$
\begin{aligned}
& \mu \cong \sum_{i=1}^{N} \widehat{y}\left(v_{i}\right) \\
& D+\mu^{2} \cong \sum_{i=1}^{N} \widehat{y}^{2}\left(v_{i}\right) \\
& D_{x}+\mu^{2} \cong \sum_{i=1}^{N} \hat{y}\left(v_{i}\right) \hat{y}\left(x_{i}, z_{i}\right)
\end{aligned}
$$

where $\mu$ is the mean value, $D$ is the total variance computed using $N$ samples of the function, and $D_{\mathrm{x}}$ is the single parameter variance due to parameter $x$. Before using Eq. (5.1) it is important to point out a notation change. As before, $v_{i}$ refers to the $i^{\text {th }}$ sample of the 
parameter vector but $x_{\mathrm{i}}$ is a parameter within $v_{i}$ about which the variance is being evaluated and $z_{\mathrm{i}}$ are all other parameters that comprise $v_{i}$ not including $x_{\mathrm{i}}$. To properly evaluate Eq. (5.1), one needs at least $2 N$ function (output) evaluations; one where all variables are randomly sampled, and a second set where all but $x_{\mathrm{i}}$ are re-sampled. With this information the $1^{\text {st }}$ index in Sobol is computed as $S_{x}=D_{x} / D$. Of course, this is only one of the $m$ possible factors. Incidentally, Eq. (5.1) is easily extended to study two or more parameter (factor) interaction, as described in [27], simply by adjusting the number of parameter that gets resampled.

A final note on ANOVA using Sobol's approach is regards convergence of the variance estimates. Although Ref. 27 discussed the asymptotic behavior of the variance estimates using Eq. (5.1), for cases where a surrogate model is used instead of LS-DYNA, the variance estimates are only as good as our surrogate model. Nonetheless, this approach provides an excellent way to rank variable importance when only a limited number of LS-DYNA runs are possible.

\section{Computational Framework}

To conduct this statistical study, it is preferable to automate the generation of LS-DYNA simulations with different parameter values. For this work the computational framework described in [32] and [33] is used based on the commercially available MATLAB ${ }^{1}$ program. MATLAB scripts are used to modify the LS-DYNA keyword input file automatically, to update the parameter values from a known distribution, to control execution of LS-DYNA, and to read LS-DYNA output files. Afterwards, results are stored within the MATLAB environment to conduct ANOVA and optimization studies. Although comparable response surface and variance tools are also available in the LS-Opt [17,34], processing of the time history data with the existing MATLAB toolbox provides more freedom.

As mentioned earlier, a companion set of LS-DYNA solutions while varying 4 parameters was created manually, outside the aforementioned computational approach, using a heuristic sampling approach to prescribe parameter values, to execute LS-DYNA, and to create a solution set. These results are also loaded into MATLAB for analysis and are presented later in the paper. 


\section{Discussion of Results}

Numerical results are discussed in sections to simplify the presentation and to highlight specific issues associated with the various aspects of the problem. First, results for response surface (RS) models are presented along with examples of LS-DYNA time history predictions using the surrogate model. Following this, results using conventional sensitivity analysis are presented. In contrast, results from variance analysis using the approach developed by Sobol are discussed using both the 4 and 7 parameter surrogate models. Finally, the RS model is used to create contours of constant Brinkley values as a function of impact conditions.

\subsection{Response Surface Modeling Results}

Before RS models are developed, a database must be created with solutions computed using LS-DYNA. For this, two independent efforts took place that produced two databases. One database contains results for the case when 4 parameters (1- vertical velocity $V_{x}$, 2horizontal velocity $V_{z}$, 3- capsule pitch angle $\theta_{y}$, and 4- wave slope $\psi_{y}$ ) are varied manually (i.e. heuristic sampling), and the second approach were 7 parameters (1- vertical velocity $V_{x}$, 2- horizontal velocity $V_{z}$, 3- capsule pitch angle $\theta_{y}$, 4- capsule yaw angle $\theta_{z}$, 5- capsule roll angle $\theta_{x}$, 6- Wave slope $\psi_{y}$, and 7- Wave direction $\psi_{x}$ ) were varied using a combination of sampling techniques which included Halton-leap, D-Optimal, and manual. Each LS-DYNA time history output file is read into MATLAB and stored for post-processing. Because the initial conditions in the LS-DYNA input key files are set using different logic, the two databases cannot be easily combined into one.

Figure 4 shows results from 231 LS-DYNA runs while varying 7 parameters. To keep the amount of data manageable, only 0.2 seconds of simulation time is created and stored, which is sufficient to capture the primary impact pulse. At the top plot of Fig. 4a is a summary of the time it takes to reach peak acceleration upon impact. This plot is part of the initial screening of the database because those cases where the peak time equals 0.2 seconds correspond to cases where the capsule is either skimming over the water or cases where the horizontal speed is so high that the capsule does not hit the water within 0.2 seconds. Fig. 4b shows the resultant maximum acceleration norm in units of gs, and Fig. 4c shows estimates for the Brinkley index, computed from predicted local accelerations at the crew location 5. 
Cases where Brinkley values are greater or equal to 1 correspond to landing conditions with probabilities of injuries.

Once a database is constructed, RS models are created to predict the time responses as a function of the input parameters. Figure 5 shows an example of predictions using Kriging response surface to predict velocities; solid blue lines correspond to two LS-DYNA solutions and the solid green lines correspond to response surface predictions. Admittedly, this plot is only a qualitative comparison of the ability of the surrogate model to predict the time responses but it shows that the response prediction is within the LS-DYNA bounds.

A more quantitative comparison illustrating the adequacy of the RS model is shown in Figs. $6 a$ and 6b where two LS-DYNA solutions are removed from the 4-parameter database and time histories are predicted with the resulting RS model. The parameter vector removed for each case is shown with the figure. Figure 6a and 5b shows LS-DYNA results in solid blue and RS predictions in dashed green. In Figure 6a the error difference between LS-DYNA and the RS model is 0.002; whereas, in Fig. 6b, after removing a different parameter set, the error difference of 0.03. Although, these are excellent results for these two cases, as is often the case with nonlinear problems, there might be regions of the solution space where the interpolation is not as good.

A note regarding the use of Kriging and/or ERBF is in order. Although both approaches provide excellent and comparable results, in cases like our 4-parameter case where sampling of the parameter space is heuristic, Kriging has problems when the sampling produces ill conditioned $R\left(v_{i}, v_{j}\right)$ in Eq.(3.1). Results with the 4-parameter database are obtained exclusively using ERBF, but with the 7-parameters database, both Kriging and ERBF provide practically identical results.

\subsection{Conventional Sensitivity Analysis of Brinkley Index}

A standard approach in design is to perform a parameter study, in which one parameter at a time is varied while the other parameters are fixed. This approach is sometimes useful to show the sensitivity of responses to a given parameter. However, it is highly dependent on the selection of the nominal values. As shown in Fig. 7, the Brinkley index is a non-linear function of the relative pitch angle (capsule pitch angle minus wave slope). In addition, the shape of the Brinkley index curve is different at different combinations of tangential and 
normal velocity. Clearly, a simple polynomial curve fits will not be able to predict the Brinkley index for Orion; more advanced response surface methods must be used.

\subsection{Analysis of Variance}

A more general approach to study the sensitivity of nonlinear system responses to parameter changes is the approach developed by Sobol. Normally, Sobol indices are computed by direct evaluation of the function values (i.e. peak accelerations, Brinkley values, etc) as the parameters are varied. For example, if one needs to evaluate the sensitivity of the peak acceleration to variations in pitch angle, LS-DYNA would have to be executed multiple times as required by Eq. (5.1). This approach is time consuming with models whose simulation times are between 12 to 24 hours. Instead, the RS model is used to provide predictions for the Sobol formula.

To aid in understanding the process, Fig. 8 shows a notional chart where the abscissa and ordinate contains Brinkley values for two crew locations 1 and 2. Each dot in the chart corresponds to a possible LS-DYNA solution when all the parameters are varied. The outer blue circle represents to the total variance, whereas the single parameter contribution is depicted using the inner black circle. Finally, the ratio of these two variances provides for a direct measure of the parameter importance.

\subsection{Four Parameter Variance Analysis}

To demonstrate the approach for the capsule impacting the water, the 4-parameter response surface model is used to estimate the parameter variance using Eq. (5.1). Figure 9 shows the fractional contribution from each parameter variation to the total variance of the Brinkley index. This fractional contribution is stacked as a percentage of the total variance (normalized to 1) observed at each of the six crew locations. Colors are used to distinguish among the different parameters, as indicated by the color-bar. Across the top of Fig. 9 are the computed mean values for the Brinkley index at each crew location. After reviewing Fig. 9, it is clear that the vertical velocity $V_{x}$ is the most important parameter followed by the wave slope $\psi_{\mathrm{x}}$. 


\subsection{Seven Parameter Variance Analysis}

In contrast to the variance results using the 4-parameter surrogate, Fig. 10 shows the results using a 7-parameter model. The results are significantly different from the 4-parameter case and show that in this case both vertical and horizontal velocities play a significant role along with the wave slope. After further review, it is observed that yaw $\theta_{z}$ and pitch $\theta_{y}$ show an increased contribution to the variance, when compared to the 4 parameter results, and significantly change the capsule behavior on impact.

To better understand these results, it is important to highlight the difference between the 4parameter and 7-parameter databases. Because these were two independent efforts, the LSDYNA initial condition set up is different. The main difference is that in the 4-parameter database, the capsule is always placed such that the closest distance from the capsule to the water is the same. Hence, during free fall, the capsule always travels the same distance. However, in the 7-parameter database this is not the case. Thus results need to be adjusted based on impact attitude and speed as opposed to the initial conditions in the LS-DYNA key file.

\subsection{Brinkley Contours}

Water impact analysis is aimed at estimating the risk of injury to the crew upon landing. For this, the Brinkley index is an industry accepted criteria to assess the risk of injury using local acceleration responses at the crew location. If only global outputs are available, the method discussed in Appendix A to compute the local accelerations should be used. This method is used with the 7-parameter database.

Brinkley contours are created from the local acceleration for the 4-parameter database and the 7-parameter database and compared as a function of the normal and tangential velocity on the impact plane. By using the normal and tangential velocities, as opposed to the initial velocities prescribed in the LS-DYNA key file, results for the two databases can now be compared. Figure 11 shows Brinkley contours at crew location 5 obtained with the 4parameter database shown in Fig. 11a (146 LS-DYNA runs) and the 7-parameter database (239 LS-DYNA runs) shown in Fig. 11b. Although there are some visible differences in the contour curvature and Brinkley values near the extreme velocities, as more LS-DYNA solutions are added to the 7-parameter database, results should move closer to those obtained 
with the 4-parameter data. This is because the 7-parameter RS model requires more LSDYNA solutions to properly capture the behavior of the system when compared to the 4parameter model. Unfortunately the question of how many LS-DYNA runs are needed does not have a simple answer. At best, users are encouraged to add solutions in those critical regions to see if there are significant changes in the results.

Brinkley contours provide engineers with a portrait of those conditions that could cause injuries. To perform this assessment using a conventional approach, one would need to compute enough LS-DYNA solutions to populate the contour. However, this approach would only give the user a limited view of the functional relationship for the particular combination of variables. In contrast, by using the RS models, a more complete portrait of Brinkley values can be generated in which all the parameters are considered. For example, Figure 12 shows the Brinkley contours at crew location 5 for pitch angles of -28 degrees Fig. 12a (nominal), -18 degrees Fig. 12b, and -38 degree Fig. 12c. Aside from the fundamental differences in the curvatures, it should be apparent that at -18 degrees pitch there are many normal/tangential velocities that result in Brinkley values of 1. In contrast, results for the case when the wave slope is varied instead between -30 and 30 degrees (with the pitch angle held at -28 degrees) resulted in the Brinkley contours shown in Fig. 13. After examining Figs. 12 and 13, it clear that changes in the capsule pitch angles are not equivalent to changes in the wave slope angle. For completeness Figs. 12 and 13 show the computed impact angles, as defined in Appendix $\mathrm{B}$, along with both pitch and the wave slope angles. Note that for a wave slope of 0 and a nominal capsule pitch angle of 28 degrees, the relative impact angle (angle between the water plane normal and capsule impact plane normal) is 28 degrees. On the other hand, a 30 degrees wave slope corresponds to a 58 degrees impact angle. When studying Fig. 13, remember that the independent variables are the initial vertical and horizontal velocities and not the tangential and normal velocities. Hence, the normal and tangential velocities are derived quantities and therefore certain values are outside the range.

\subsection{Estimation of Critical Impact Conditions}

A more important use of the RS model is to recover critical landing conditions. In Fig. 14 is an illustration showing Brinkley contours with a fictitious probability distribution function superimposed for the normal velocity. If the probability distribution function is known, then it 
is possible to compute the most probable set of normal velocities that would cause Brinkley to exceed 1. This kind of information is precisely what the first and second order reliability methods provide.

To demonstrate the use of RS models to estimate critical landing conditions, the 4parameter RS model is used with a genetic optimization algorithm to compute critical conditions. Because parameter distribution functions are not readily available, the optimizer is instructed to look for parameter sets that produce Brinkley values of 1 . Table 4 shows one parameter set of the many sets computed by the optimizer. To verify that this is indeed a critical landing condition, LS-DYNA is executed with the parameters shown in the table and the time history from LS-DYNA is plotted in Fig. 15 in solid blue versus the prediction from the response surface model in dashed green. Although the RS prediction is not exact, it is very good, and more importantly it predicted a Brinkley index near 1.

\section{Concluding Remarks}

The paper discussed an approach that combines solutions from the commercial code LSDYNA with response surface techniques to estimate critical impact conditions of a capsule while landing on water. What makes this problem so challenging is the complexity of the water and capsule interaction, and the computational time it takes to generate enough solutions to understand the physics of the problem. Oftentimes, statistical analysis of problems this complex is not undertaken because the computational burden is significant. It is shown how to use a limited number of high fidelity LS-DYNA solutions with response surface techniques, like Extended Radial Basis Functions and Kriging, to conduct statistical analysis and to study conditions under which crew injuries are likely to occur. In addition, the approach also allows for sensitivity studies, using an approach developed by Sobol, to screen and rank parameters based on their contribution to the output.

Initial results for the capsule landing on water show that the vertical velocity and the impact plane slope are the two most important parameters. Results also showed that although certain angle combinations, e.g. capsule pitch and wave slope, produce the same impact angles, the system response and Brinkley estimates can be significantly different. 


\section{Acknowledgements}

The authors would like to thank James M. Corlis for his help during the problem definition phase, Dr. John T. Wang for providing the initial LS-DYNA water model that was the basis for the model used in the paper, and also special thanks to Gabrielle S. Snyder for her help setting up the computer cluster for the LS-DYNA runs. 


\section{References}

1. Hartwig, H.H and Schmidt, J. L.: "Summary- Apollo Earth Landing System Impact Attenuation Trade-Off Study,” North American Aviation SID 62-897, July 1962.

2. Stubbs, S.M.: "Investigation of the Skid-Rocker Landing Characteristics of Spacecraft Models," NASA TN D-1624, April 1963.

3. Benson, H.E. and West, R.B.: "Results of Two One-Quarter Scale Apollo Model Impact Tests Utilizing Different Impact Attenuation Systems,” NASA TMX-65129, January 1965.

4. "Water Impact of the Mercury Capsule Correlation of Analysis with NASA Tests," Grumman Aircraft Engineering Corporation, NASA CR-55251, December 1962.

5. Thompson, W.C.: "Dynamic Model Investigation of the Rough-Water Landing Characteristics of a Spacecraft,” NASA TN D-3774, April 1967 (Technical Film Supplement L-940).

6. Reese, T. and Rose, J.D.: “Apollo Pad Abort Land Impact Tests at Kennedy Space Center,” NASA TM X-64365, April 1969.

7. McCullough, J. E. and Lands, J. F. Jr.: “Apollo Command Module Land-Impact Tests,” NASA TN D-6979, October 1972.

8. Baker, W. E., Westine, P. S., Garze, L. R., Hunter, P. A.: "Water Impact Studies of Model Apollo Command Module,” NASA CR-114949, August 1965.

9. Baker, W.E and Westine, P.S.; "Model Tests for Structural Response of Apollo Command Module to Water Impact,” AIAA Journal of Spacecraft and Rockets, 00224650, Vol. 4 No. 2, 1967.

10. Stubbs, S.M.: "Dynamic Model Investigation of Water Pressures and Accelerations Encountered During Landings of the Apollo Spacecraft,” NASA TN D-3980, September 1967. (Technical Film Supplement L-960).

11. Stubbs, S. M Hathaway, M. E.: "Effects of Bottom-Structure Flexibility on Water Landing Loads of Apollo Spacecraft Models,” NASA TN D-5108, March 1969.

12. "Final Test Report Water Impact Test 92 Boilerplate 28 (Drop 3)", North American Aviation SID 66-35, April 1967.

13. “Final Test Report Water Impact Test 104 Spacecraft 2S-1 (Drop 2)”, North American Aviation SID 66-330A, April 1967.

14. "Study of Apollo Water Impact Final Report: Volume 4 - Comparison with Experiments,” North American Aviation, NASA CR-92022, May 1967.

15. "Apollo Applications Program Preliminary Evaluation Data - Apollo Landing and Recovery Subsystem Analyses,” North American Rockwell, SID-69-244, April 1969.

16. Brinkley, J.W., and Moser, S.E.: "Development of Acceleration Exposure Limits for Advanced Escape Systems," AGARD CP-472, April, 1989.

17. Anonymous: “LS-DYNA Keyword User’s Manual,” Version 971, Livermore Software Technology Company, Livermore, CA, August 2006.

18. Sacks, J., Schiller, S.B, and Welch, W.J.: "Design of Computer Experiments," Technometrics, February 1989, Vol. 31, No. 1

19. Mullur, A. and Messac, A.: "Extended Radial Basis Functions: More Flexible and Effective Metamodeling.” AIAA Journal, Vol., 43, No. 6, June 2005. 
20. Mullur, A. and Messac, A.: "Metamodeling Using Extended Radial Basis Functions: A Comparative Approach.” Engineering with Computers (2006) 21: 203-217 DOI 10.1007/s00366-0005-7

21. Chipperfield, A., Fleming, P. J., Pohlheim, H. and Fonseca, C. M.: "Genetic Algorithm Toolbox for use with Matlab.” Technical Report No. 512, Department of Automatic Control and Systems Engineering, University of Sheffield, 1994

22. Iman, R.J., and Shortencarier, M.J.: "A FORTRAN77 Program and User's Guide for Generation of Latin Hypercube and Random Samples for Use with Computer Models," NUREG/CR-3624, SAND83-2365, Sandia National Laboratories, Albuquerque, NM.

23. Kalagnanam. J.R, and Diwekar, U.M.:" An efficient Sampling Technique for Off-line Quality Control." Technometrics, August 1997, Vol. 39, No. 3, pp 308-319.

24. Montgomery, D.C: Design and Analysis of Experiments, $6{ }^{\text {th }}$ Edition, Copyright $(c)$ 2005 John Wiley and Sons, Inc.

25. Halton, J.H., "On the Efficiency of Certain Quasi-Random Sequences of Points in Evaluating Multi-Dimensional Integrals,” Numerische Mathematik, Vol. 2, pp. 84-90, 1960

26. Robinson, D., and Atcitty, C., "Comparison of Quasi-and Pseudo-Monte Carlo Sampling for Reliability and Uncertainty Analysis," American Institute of Aeronautics and Astronautics, A99-24882, and AIAA-99-1589.

27. Sobol, I.M., Tarantola, S., Gatelli, D., Kucherenko, S.S, and Mauntz, W., "Estimating Approximation Error When Fixing Unessential Factors in Global Sensitivity Analysis," Reliability Engineering and System Safety 92 (2007) 957-960, @ 2006 Elsevier LTD

28. Mullershon, H., and Liebsher, M, "Statistics and Non-Linear Sensitivity Analysis with LS-OPT and DSPEX.” $10^{\text {th }}$ International LS-DYNA Users Conference, pp. 4-1,4-13

29. Homma, T, and Saltelli, A, "Importance Measures in Global Sensitivity Analysis of Nonlinear Models.” Reliability Engineering and System Safety 52 (1996) 1-17, (c) 1996 Elsevier LTD.

30. Sudret, B, "Global Sensitivity Analysis Using Polynomial Chaos Expansion," Reliability Engineering and System Safety 93 (2008) 964-979, (c) 2007 Elsevier LTD

31. Stander, N. and Goel, T.: "Metamodel Sensitivity to Sampling Strategies: A Crashworthiness Design Study.” $10^{\text {th }}$ International LS-DYNA Users Conference, pp. 4-154-29.

32. Horta, L.G., Jackson, and K.E., Kellas, S: "A Computational Approach for Model Update of an LS-DYNA Energy Absorbing Cell." Proceedings of the 2008 AHS 64 Annual Forum \& Technology Display, April 29-May 1, Montreal Canada.

33. Horta, L.G. and Reaves, M.C.:" On the Application of a Response Surface Technique to Analyze Roll-Over Stability of Capsules With Air Bags Using LS-DYNA." NASA TM 2008-215117, March 2008.

34. Stander, N, Eggleston, T, Craig, K, and Roux, W: "LS-OPT User’s Manual A Design Optimization and Probabilistic Analysis Tool for the Engineering Analyst.” Version 2, Oct. 2003. 


\section{Appendix A: Estimation of Response for Arbitrary points on Rigid Body}

With the complexity associated with LS-DYNA models, particularly when modeling fluids/structure interaction, the amount of data that is created can be overwhelming. For storage purposes, it is more efficient to store only a subset of the response data if other data can be recovered from it. The ability to recover information from a subset of data is also important in those cases where engineers, after completing a set of simulations, realized that they should have monitored other points not included in the stored output. Of course, one option is to re-run all simulations to store the data for the points left out, but a more efficient way, for rigid structures, is to store the least amount of information and then compute all other information from the stored data. This approach is, in fact, possible and more efficient provided that the initial information stored is judiciously selected to allow for general 3dimensional motion to be recovered. In the following, the reader is reminded of the equations to recover the responses at arbitrary points.

Recall that from rigid body kinematics, the relative velocity of point $c$ with respect to point $m$ is given as

$$
\vec{v}_{c}-\vec{v}_{m}=\vec{v}_{c / m}=\vec{\omega} \times \vec{R}_{c / m}
$$

where $\vec{\omega}$ is the angular velocity of the body and $\vec{R}_{c / m}$ is the relative position of $c$ with respect to $m$. There are at least two ways that Eq. (11.1) can be used; 1) if the velocity of point $c$ and $m$ are known, the angular velocity of the body can be estimated knowing the body's geometry, and/or 2) if the information for an arbitrary point $d$ located at $\vec{R}_{d / m}$ is needed, given the body angular velocity, the relative velocity of $d$ is also given by Eq. (11.1) .

Similarly, if acceleration information is needed, the acceleration of point $c$ with respect to $m$ is given as

$$
\vec{A}_{c}=\vec{A}_{m}+\vec{A}_{c / m}=\vec{A}_{m}+\vec{\omega} \times \vec{v}_{c / m}+\vec{\alpha} \times \vec{R}_{c / m}
$$

where now the body angular acceleration vector $\vec{\alpha}$ is introduced. As before, depending upon what information is stored with the rigid body simulation, accelerations for points anywhere in the body are easily computed using Eq. (11.2). 
For results in the present paper, the angular position, angular velocity, and angular accelerations are all available. Hence, Eqs. (11.1) and (11.2) are used with the location of new points for estimation of acceleration values.

Although response extraction points on the body may seem futile, for cases where the location of the point at which the response is uncertain, this method provides a simple mechanism to examine the variation in the system response to establish uncertain bounds. Otherwise, new simulations would need to be executed when evaluating new points. 


\section{Appendix B: Estimation of Impact Velocities and Angles}

In this study, many response quantities are computed directly within LS-DYNA but there are others like impact angles and velocities that must be derived from the LS-DYNA outputs. In the following, the equations used to compute the impact angles and velocities are provided.

\subsection{Estimation of Impact Angles}

Consider, the capsule shown in Figure B.1. Assume that the capsule motion starts with an initial velocity and pitch orientation, falls under a gravity and impacts a water surface that is defined by a point $\vec{R}_{p}$ on the surface and its unit normal $\vec{n}_{p}$. Also consider a capsule with a moving reference frame attach to point $m$, which is located at the intersection of the capsule's center line and an imaginary plane containing the impact point $I P$. In the following, relative vector definitions will have a subscript “a/b” to indicate that it is a vector pointing from point $b$ to point $a$, i.e. making the point $b$ the reference. Vectors without an explicit reference are defined with respect to the inertial frame. Finally, if a vector quantity is used without the upper arrow, it is referring to the vector of components, for example $R_{a / b}=\left[\begin{array}{lll}x & y & z\end{array}\right]^{\prime}$.

To estimate the impact angles of a capsule with the water, first the impact point IP on the water must be located at the intersection of the capsule and water planes. With the aid of Fig. B.1 let an impact plane on the capsule be defined by its normal $\vec{n}_{c}$ and a center point $m$ located at $\vec{R}_{m}$. As mentioned earlier, the water impact plane is defined by its normal $\vec{n}_{p}$ and a point $p$ located at $\vec{R}_{p}$. Now the vector difference between point $c$ on the capsule impact plane (located at $\vec{R}_{c}$ ) and $\vec{R}_{m}$ must be orthogonal to $\vec{n}_{c}$, hence;

$$
n_{c}^{T}\left(R_{c}-R_{m}\right)=0
$$

Similarly, for an arbitrary point on the water plane IP located at $\vec{R}_{I P}$, the vector difference between it and $\vec{R}_{p}$ must orthogonal to $\vec{n}_{p}$ and thus satisfies the equation

$$
n_{p}^{T}\left(R_{I P}-R_{p}\right)=0
$$

The plane equations (12.1) and (12.2) are written in terms of vector differences of two arbitrary points on each of the respective planes. In addition to these two equations, the 
impact point, if it exists, is located along the direction $\vec{B}=\vec{n}_{c} \times \vec{n}_{p}$, i.e., orthogonal to both normal vectors. For cases where both planes (capsule and water) are rectangular, $\vec{B}$ points along the intersection line, but, if one of the impact planes is circular (which is our case), the intersection line reduces to a single point.

Because the capsule impact plane normal $\vec{n}_{c}$ is known or can be estimated from the capsule displacements, this normal can be used to express any point on the capsule impact plane as

$$
f=U q=R_{c}-R_{m}
$$

where the matrix $U$ is an $\Re^{3 \times 2}$ constructed using the basis vectors from null space of $n_{c}$, i.e., any orthogonal vector set perpendicular to $n_{c}$; whereas $q$ is an $\mathfrak{R}^{2 \times 1}$ vector of arbitrary parameters to be computed later. Using the same logic, any point on the impact plane can be written as

$$
w=G z=R_{I P}-R_{p}
$$

where the matrix $G$ is $\mathfrak{R}^{3 \times 2}$ constructed from the null space of $n_{p}$ and $z$ is an $\mathfrak{R}^{2 \times 1}$ vector of arbitrary parameters. In Eqs. (12.3) and (12.4) each of the arbitrary vectors $q$ and $z$ has dimensions reduced from 3 to 2 .

Since $\vec{R}_{I P}$ is the impact point, the following vector summation must hold

$$
R_{m}+f=R_{I P}
$$

and

$$
R_{p}+W=R_{I P}
$$

Because the impact point has no projection along $\vec{B}$, this fact is represented as

$$
B^{T}\left(R_{m}+f\right)=0
$$

or equivalently,

$$
B^{T}\left(R_{p}+w\right)=0
$$

Combining Eqs. (12.3), (12.4), (12.5), (12.6), and (12.8), yields the following set of 4 equations and four unknowns

$$
\left[\begin{array}{cc}
U & G \\
0 & B^{T} G
\end{array}\right]\left\{\begin{array}{l}
q \\
z
\end{array}\right\}=\left\{\begin{array}{c}
-R_{m}+R_{p} \\
-B^{T} R_{p}
\end{array}\right\}
$$


Solving for $q$ and $z$, and substituting back into (12.5)or (12.6) provides the coordinates of the impact point $\vec{R}_{I P}$.

Once the impact point location is known, the impact angles can be easily computed. Impact angle $\theta$, which is independent of the impact point, is computed using the normal vectors as

$$
\theta=\cos ^{-1}\left(n_{c}^{T} n_{p}\right)
$$

Impact angle $\psi$ is the angle between a traveling direction $\vec{R}_{c / m}$ and the impact point $\vec{R}_{I P / m}$ (note that both vectors are now defined with respect to $m$ ). Using these definitions the angle is defined as

$$
\psi=\cos ^{-1}\left(\frac{R_{I P / m}^{T} R_{c / m}}{\left|R_{I P / m}\right|\left|R_{c / m}\right|}\right)
$$

As is always the case when defining angles, there is arbitrariness in the way the normals are defined and, as such, these definitions must be adjusted to conform to a particular sign convention. 


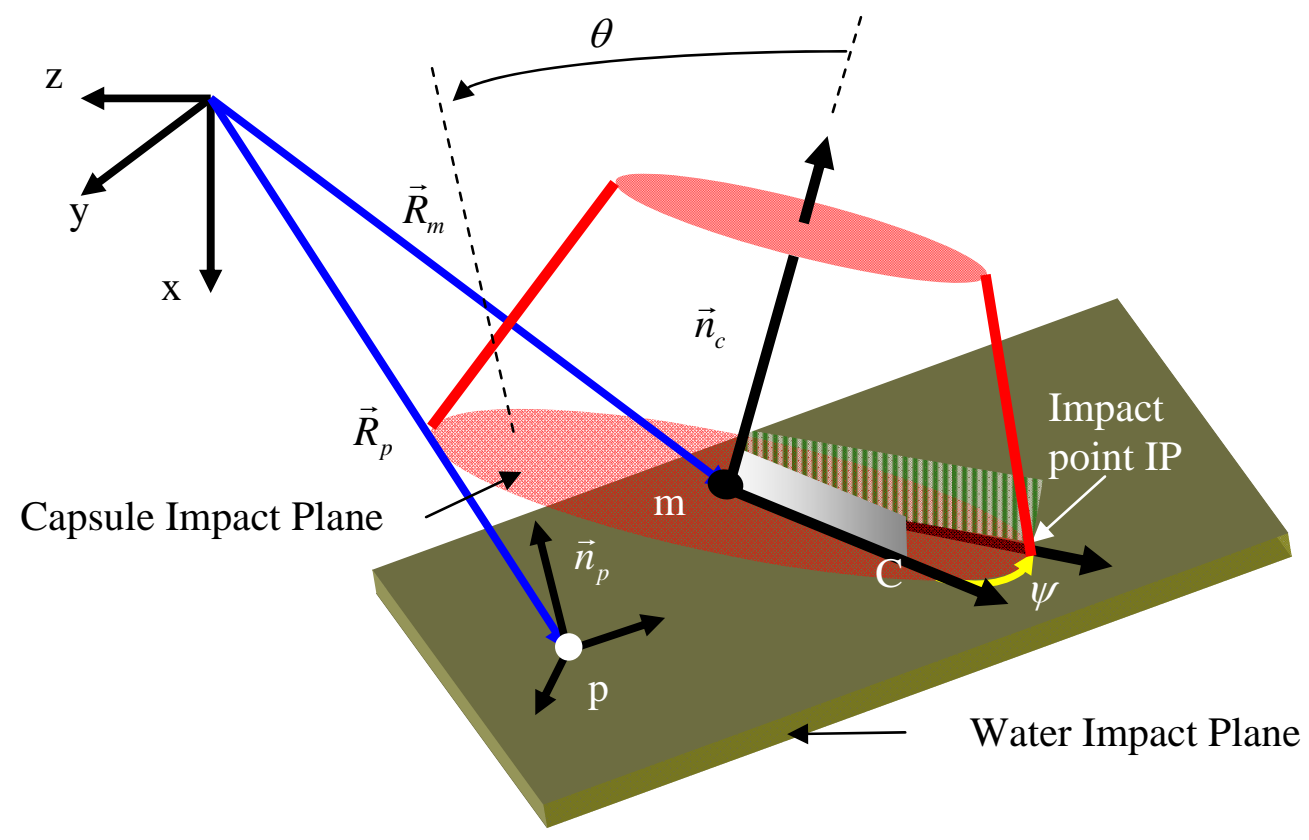

Fig. B.1 Capsule and water plane parameter description

\subsection{Estimation of Impact Velocities}

Impact plane location and orientation is provided in terms of a point on the plane and its unit normal vector, but to compute impact velocities based on the capsule orientation and direction of travel, it is necessary to define the impact plane in terms of a local coordinate system. Using the impact plane normal unit vector $\vec{n}_{p}$ a set of orthogonal axes can be defined in terms of the null space of $\vec{n}_{p}$. If the two bases vectors for the null space are represented as $G=\left[\begin{array}{ll}g_{1} & g_{2}\end{array}\right]$, one can use the vector $\vec{B}=\vec{n}_{c} \times \vec{n}_{p}$, which is normal to both planes, as one of the three components of the impact plane coordinate system. If the projection of the vector $\vec{B}$ along $g_{1}$ and $g_{2}$ is given as $\alpha_{1}=g_{1} \circ B$ and $\alpha_{2}=g_{2} \circ B$ (where $\circ$ means dot product), the traveling direction is defined as that for which $\alpha_{i}$ is equal to zero. Since our bases vectors are not necessarily aligned with any particular axis, one can compute the component along the tangential direction (traveling direction) as one of these three vectors; 


$$
\begin{array}{ll}
\vec{l}=g_{1}-\frac{\left(g_{1} \circ B\right) g_{1}}{\left|g_{1}\right||B|}, & \text { if } \alpha_{1} \neq 0, \alpha_{1} \neq 1, \text { or } \\
\vec{l}=\frac{g_{1}}{\left|g_{1}\right|}, & \text { if } \alpha_{1} \neq 0, \alpha_{1}=1, \text { or } \\
\vec{l}=g_{2}-\frac{\left(g_{2} \circ B\right) g_{2}}{\left|g_{2}\right||B|}, & \text { if } \alpha_{1}=0,
\end{array}
$$

Using $\vec{w}=-\vec{n}_{p} \times \vec{l}$ as the third component of the coordinate system, the coordinate system is now defined by the triad $\left[\begin{array}{lll}-\vec{n}_{p} & \vec{l} & \vec{w}\end{array}\right]$. The negative sign on the impact plane normal is there to accommodate a particular sign convention.

Finally, using these direction cosines, velocities along the normal and tangential components are computed using the dot product of the capsule velocities on impact and the impact plane triad. 
Table 1 Mass and inertial properties of capsule

\begin{tabular}{|c|c|c|c|}
\hline Mass, $\mathbf{l b}_{\mathbf{f}} \mathbf{s}^{\mathbf{2}} / \mathbf{i n}$ & $\mathbf{I}_{\mathbf{X X}}, \mathbf{l b}_{\mathbf{f}} \mathbf{s}^{\mathbf{2}}$ in & $\mathbf{I}_{\mathbf{Y Y}}, \mathbf{l b}_{\mathbf{f}} \mathbf{s}^{\mathbf{2}}$ in & $\mathbf{I}_{\mathbf{Z Z}}, \mathbf{l b}_{\mathbf{f}} \mathbf{s}^{\mathbf{2}}$ in \\
\hline \hline 49.0536 & 216,252 & 136,104 & 177,684 \\
\hline
\end{tabular}

Table 2. Parameter definitions

\begin{tabular}{|c|c|c|c|c|}
\hline $\begin{array}{c}\text { Parameter } \\
\text { Number }\end{array}$ & $\begin{array}{c}\text { Parameter } \\
\text { Name }\end{array}$ & $\begin{array}{l}\text { Upper } \\
\text { Bound }\end{array}$ & Nominal & $\begin{array}{l}\text { Lower } \\
\text { Bound }\end{array}$ \\
\hline 1 & $\begin{array}{c}\text { Vertical Speed } \\
V_{x}(\mathrm{in} / \mathrm{sec})\end{array}$ & 600 & 360 & 120 \\
\hline 2 & $\begin{array}{c}\text { Horizontal } \\
\text { Speed } V_{z}(\text { in/sec })\end{array}$ & 0 & -480 & -960 \\
\hline 3 & $\begin{array}{l}\text { Pitch Rotation } \\
\qquad \theta_{\mathrm{y}}(\mathrm{deg})\end{array}$ & -18 & -28 & -38 \\
\hline 4 & $\begin{array}{c}\text { Yaw Rotation } \\
\theta_{z}(\mathrm{deg})\end{array}$ & 10 & 0 & -10 \\
\hline 5 & $\begin{array}{l}\text { Roll Rotation } \\
\theta_{x}(\mathrm{deg})\end{array}$ & 30 & 0 & -30 \\
\hline 6 & $\begin{array}{c}\text { Wave Slope } \\
\psi_{\mathrm{x}} \text { (deg) }\end{array}$ & 30 & 0 & -30 \\
\hline 7 & $\begin{array}{c}\text { Wave Roll } \\
\psi_{\mathrm{y}}(\mathrm{deg})\end{array}$ & 30 & 0 & -30 \\
\hline
\end{tabular}


Table 3. Solid element dimensions for 4 mesh cases

\begin{tabular}{|c|c|c|c|c|}
\hline $\begin{array}{c}\text { Mesh } \\
\text { Case }\end{array}$ & $\begin{array}{c}\text { Far-Field } \\
\text { Air, in. }\end{array}$ & $\begin{array}{c}\text { Interface } \\
\text { Air, in. }\end{array}$ & $\begin{array}{c}\text { Interface } \\
\text { Water, in. }\end{array}$ & $\begin{array}{c}\text { Far-Field } \\
\text { Water, in. }\end{array}$ \\
\hline \hline 1 & 4 & 4 & 4 & 4 \\
\hline 2 & 16 & 16 & 4 & 4 \\
\hline 3 & 4 & 1 & 1 & 4 \\
\hline 4 & 4 & 4 & 1 & 4 \\
\hline
\end{tabular}

Table 4. Example of estimated critical landing parameters

\begin{tabular}{|c|c|c|}
\hline Parameter & Nominal & $\begin{array}{c}\text { Critical } \\
\text { Landing } \\
\text { Condition }\end{array}$ \\
\hline \hline Vertical Speed & 360 & 501.9 \\
$V_{x}$ (in/sec) & -480 & -47.4 \\
Horizontal Speed & & -26 \\
$V_{z}$ (in/sec) & -28 & 0 \\
Pitch Rotation & & \\
$\theta_{\mathrm{y}}$ (deg) & 0 & 0 \\
Yaw Rotation & & \\
$\theta_{\mathrm{z}}$ (deg) & 0 & -5 \\
Roll Rotation & 0 & \\
$\theta_{\mathrm{x}}$ (deg) & & \\
Wave Slope & & \\
$\psi_{\mathrm{x}}$ (deg) & & \\
Wave Roll & & \\
$\psi_{\mathrm{y}}$ (deg) & 0 & \\
\hline
\end{tabular}




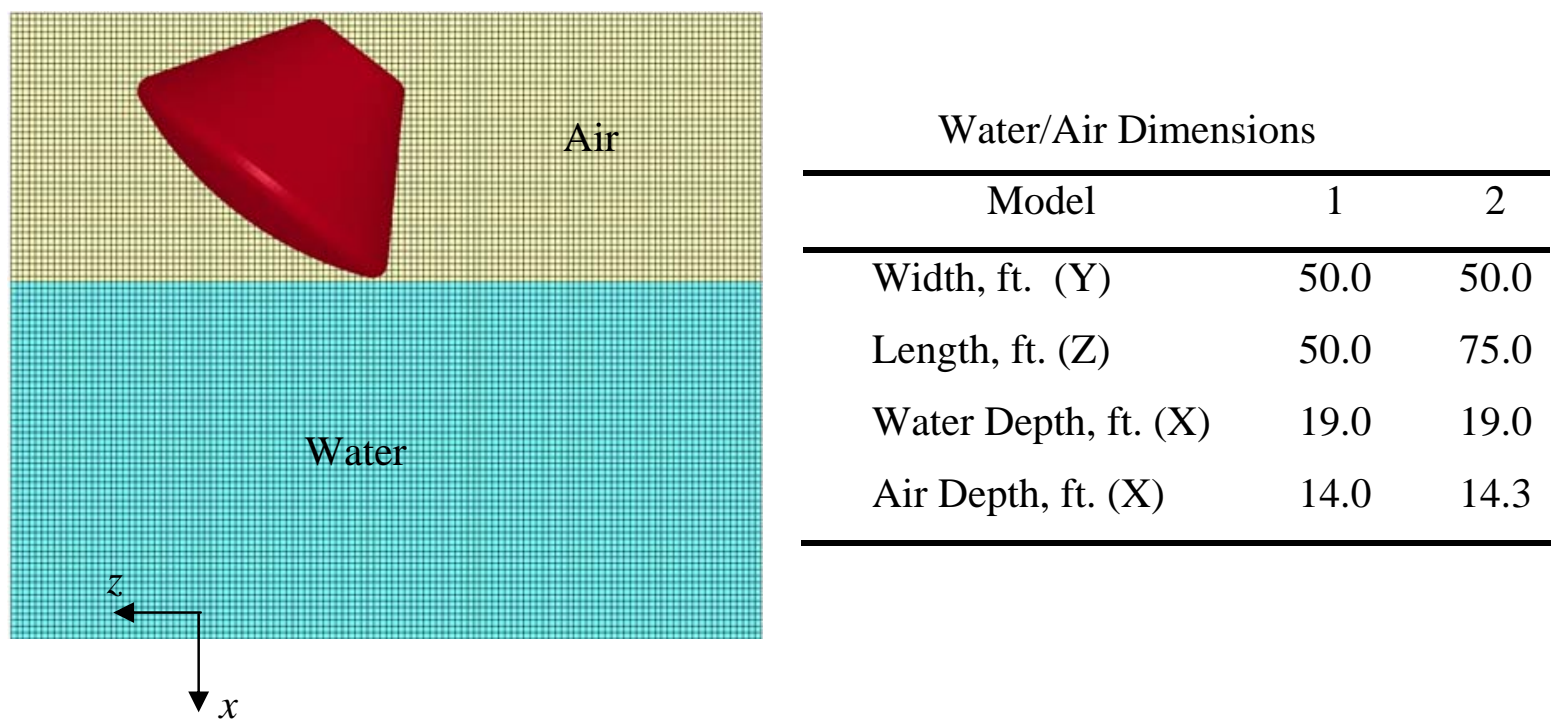

Fig. 1 Model dimensions.

Toe-in

(Negative Pitch

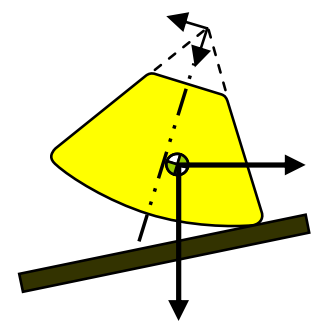

Uphill

(Positive Slope Angle)

Direction of Forward Velocity
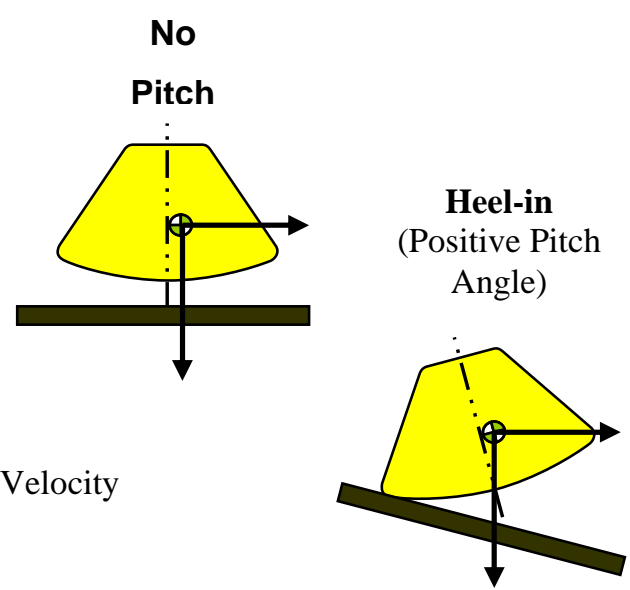

Downhill

(Negative Slope)
Heel-in

(Positive Pitch Angle)

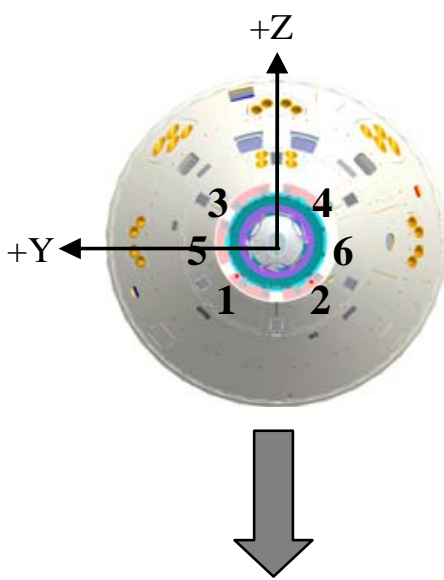

Direction of

Forward Velocity

Fig. 2 Capsule and water orientation definitions with approximate crew location seating. 


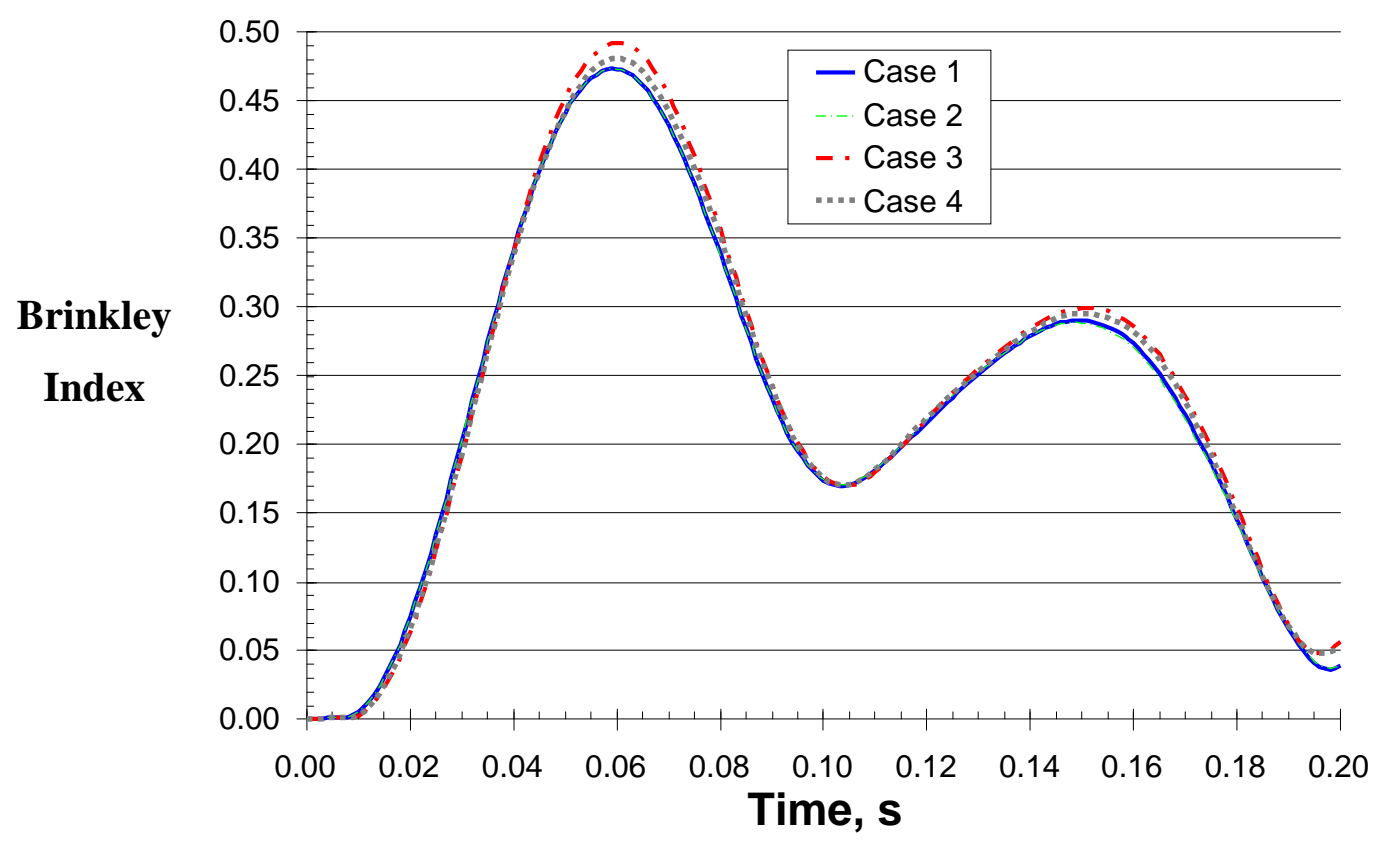

Fig. 3 Mesh sensitivity results using Brinkley Index.

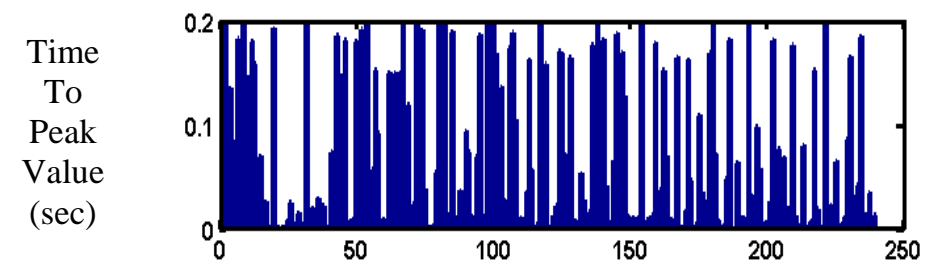

4a) time to peak acceleration

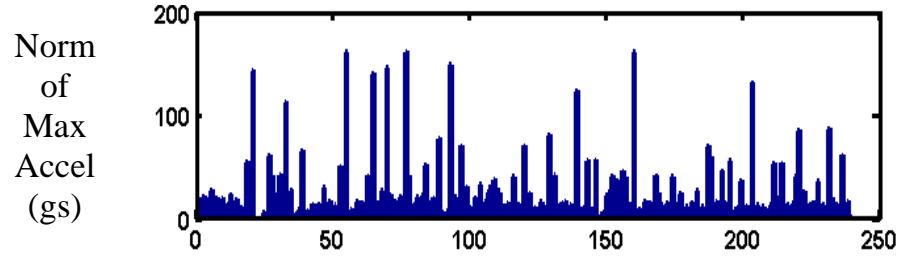

4b) norm of resultant acceleration

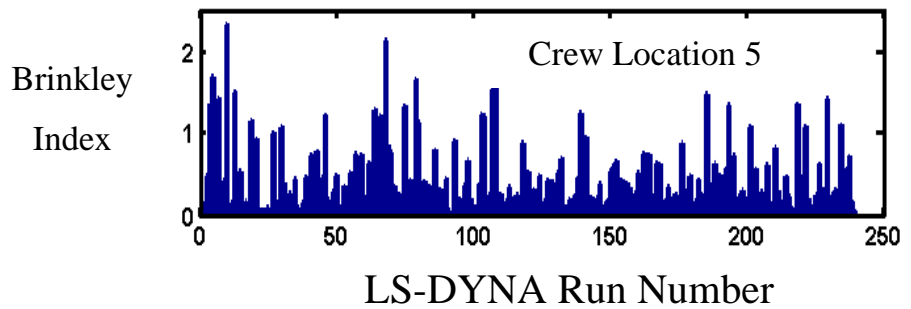

4c) peak Brinkley value

Fig. 4 Summary of LS-DYNA response for 7 parameter database. 


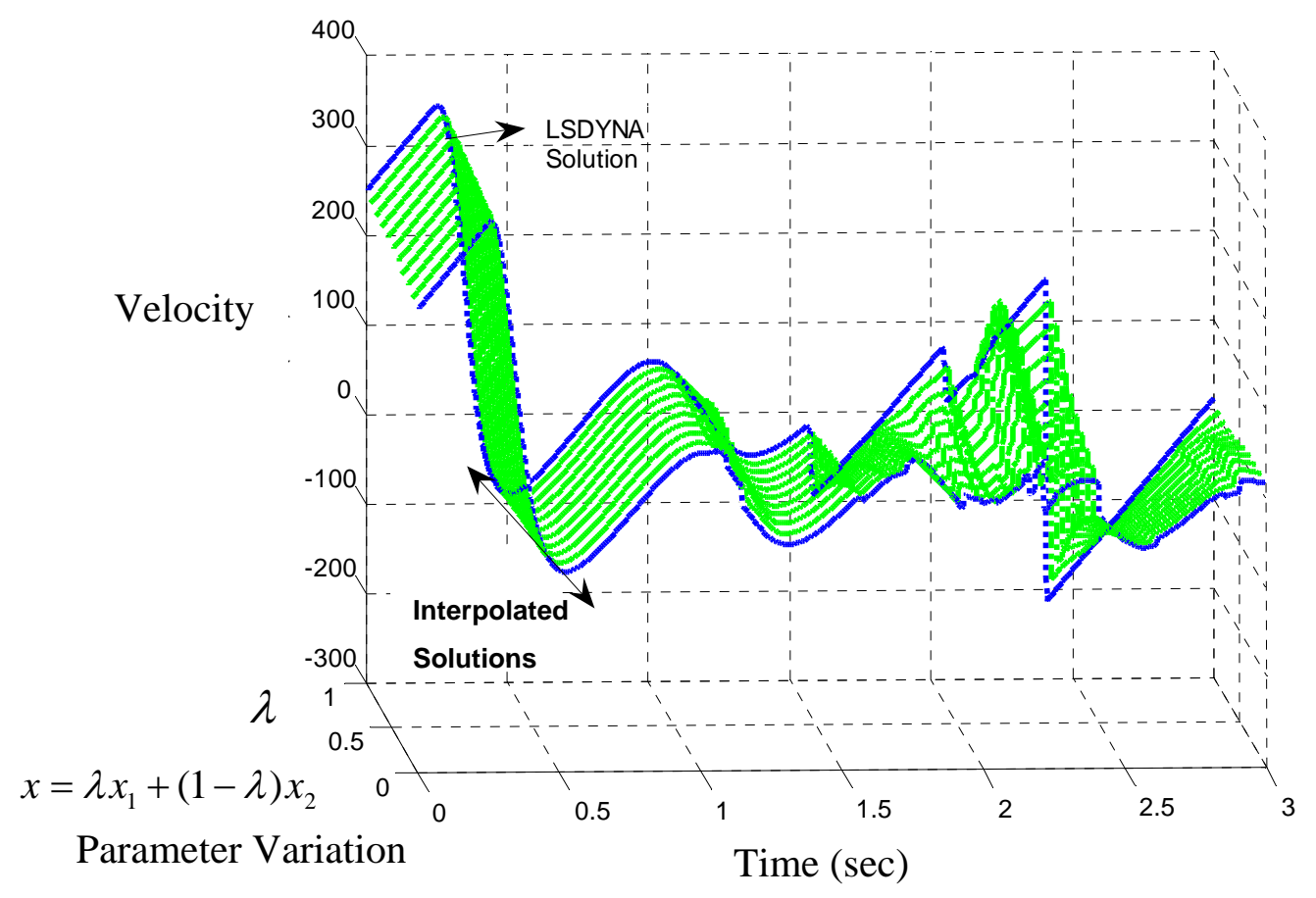

Fig. 5 Example of response surface predictions of LS-DYNA time. 


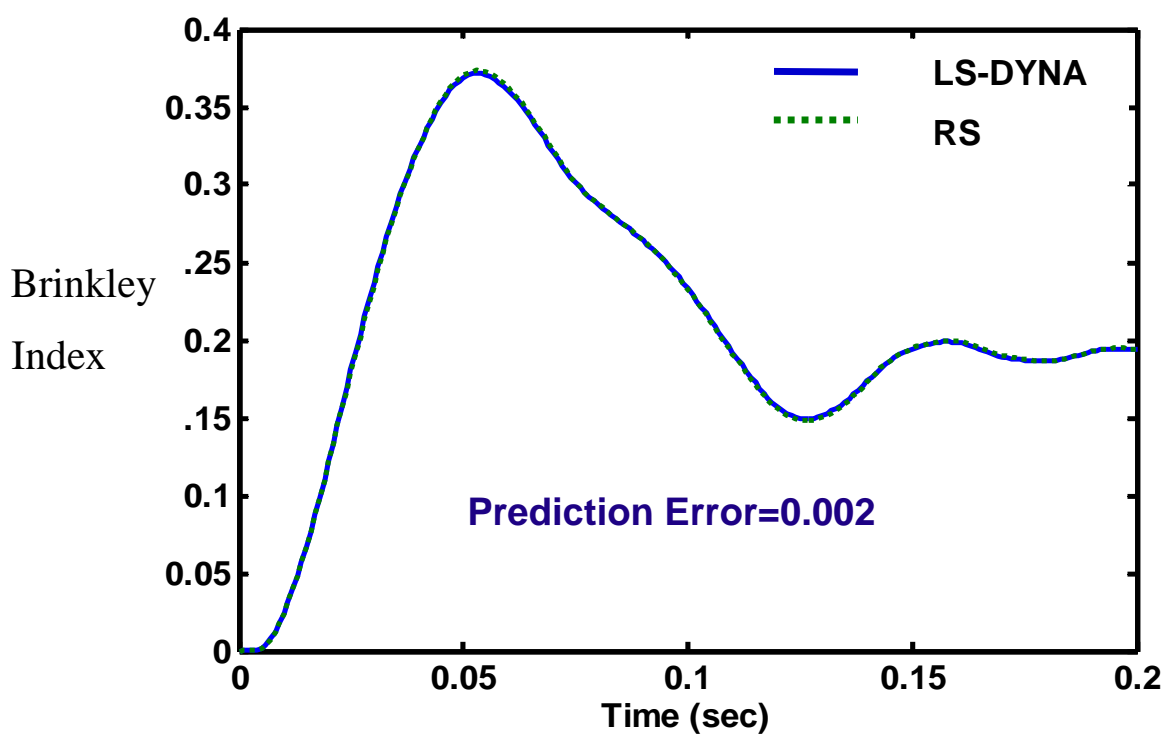

a) Parameters removed $P=[360 \mathrm{in} / \mathrm{s},-480 \mathrm{in} / \mathrm{s},-28 \mathrm{deg}, 0 \mathrm{deg}]$

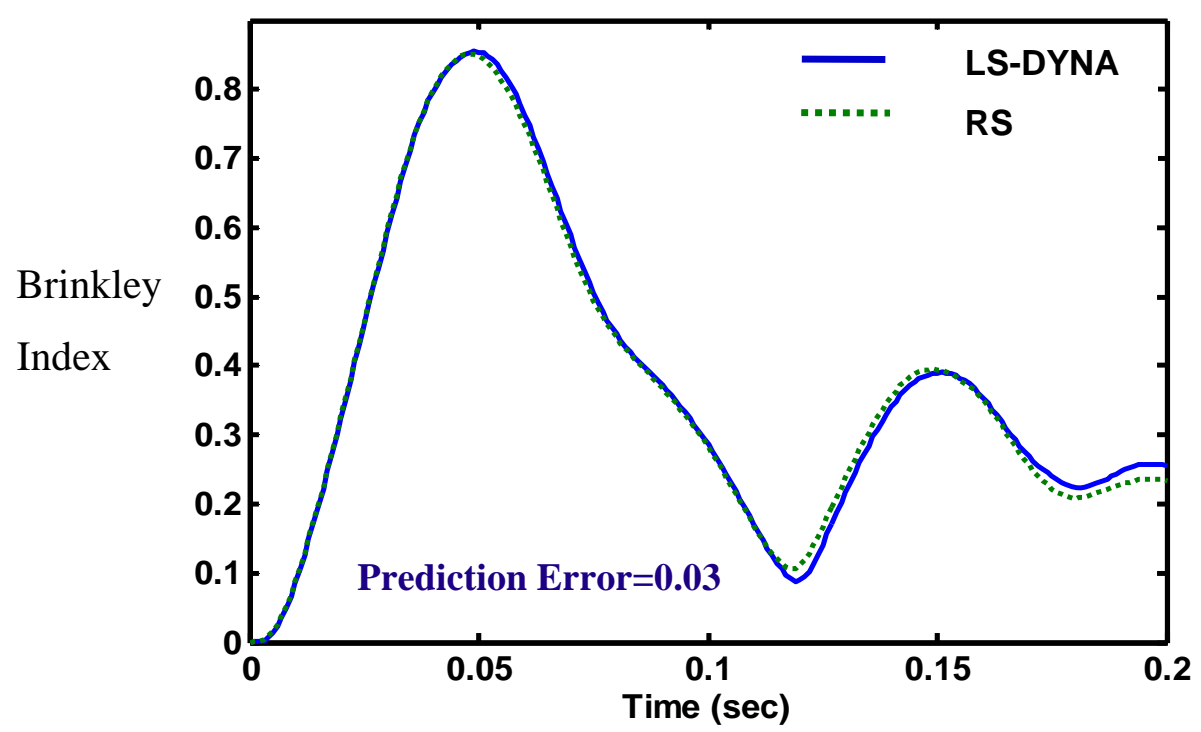

b) Parameters removed $P=[540 \mathrm{in} / \mathrm{s},-480 \mathrm{in} / \mathrm{s},-28 \mathrm{deg}, 0 \mathrm{deg}]$

Fig. 6 Verification of response surface and LS-DYNA time history predictions at crew location 4 after removing solution from the response surface. fitting 


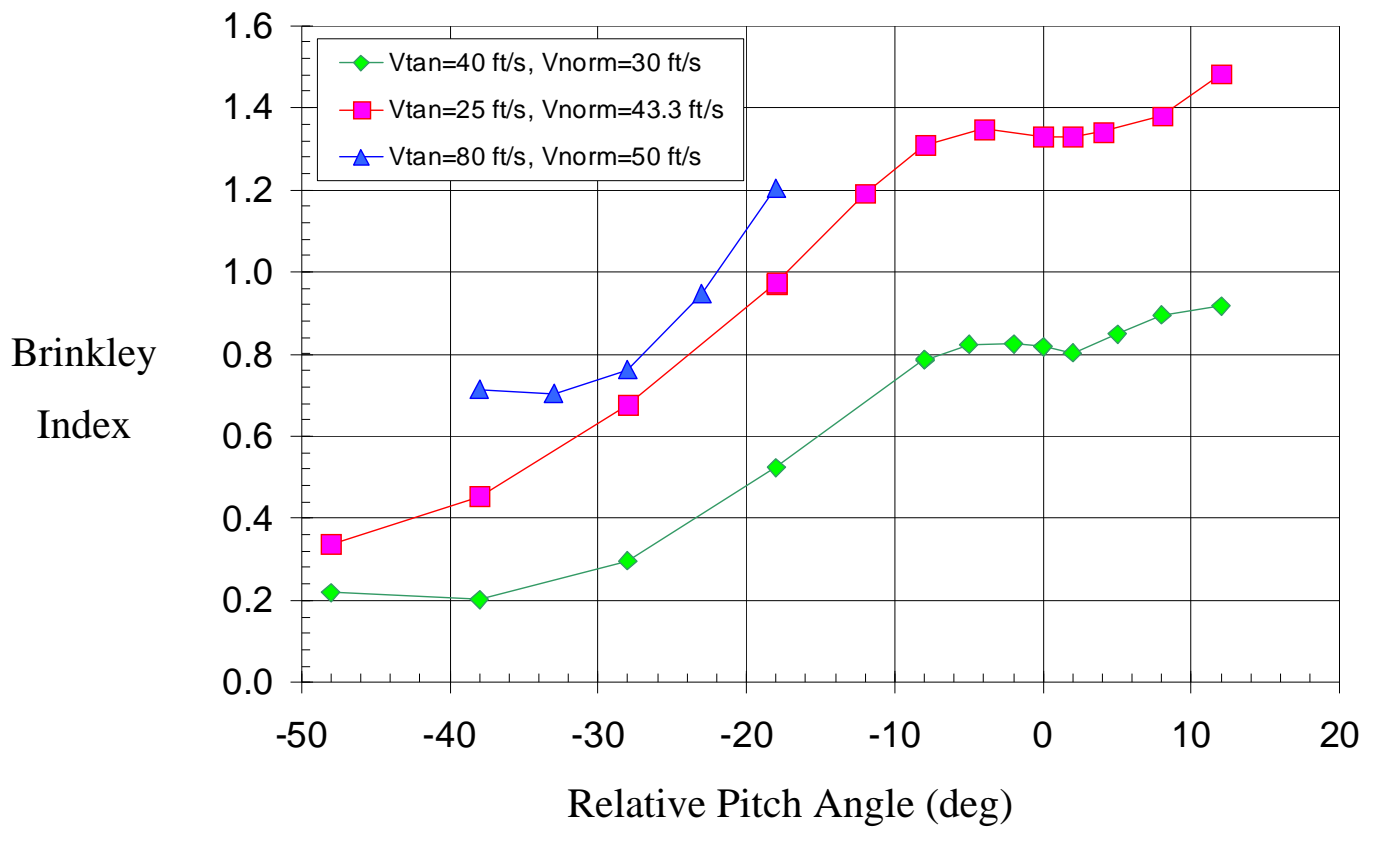

Fig. 7 Single variable sensitivity analysis.

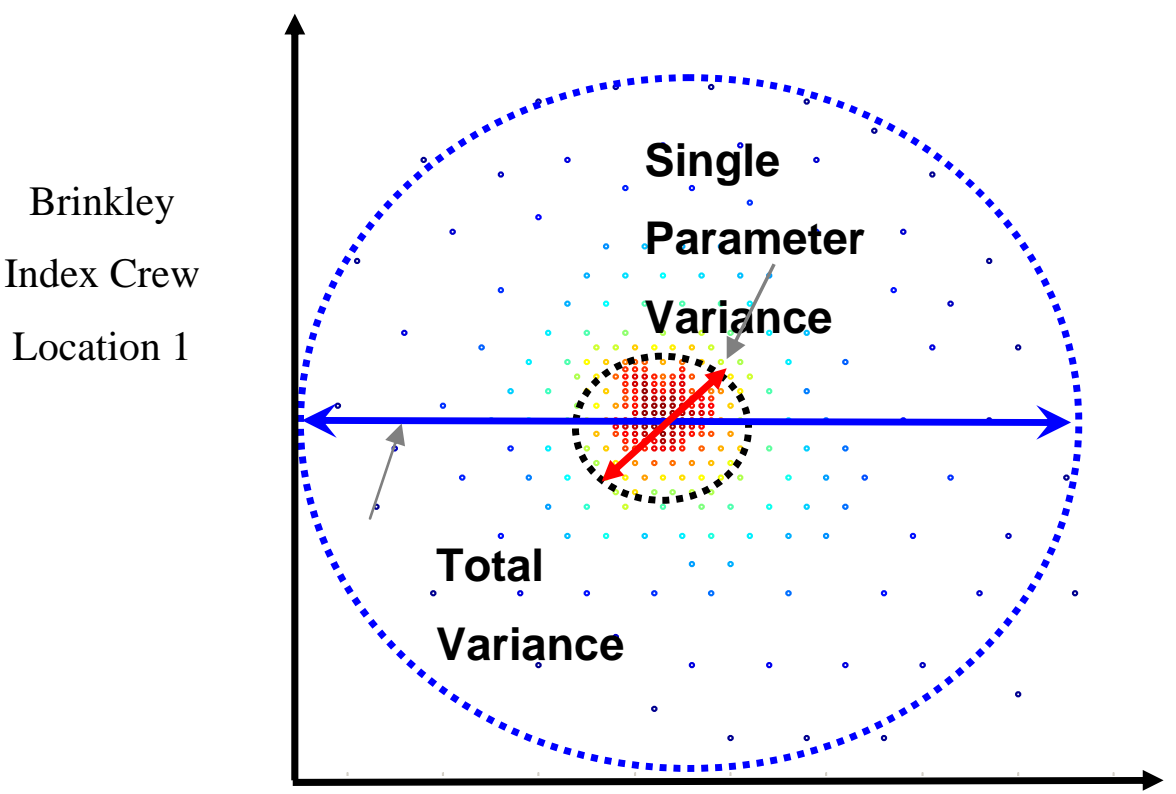

Brinkley Index Crew Location 2

Fig. 8 Example of analysis of variance. 
Brinkley Index Mean Values

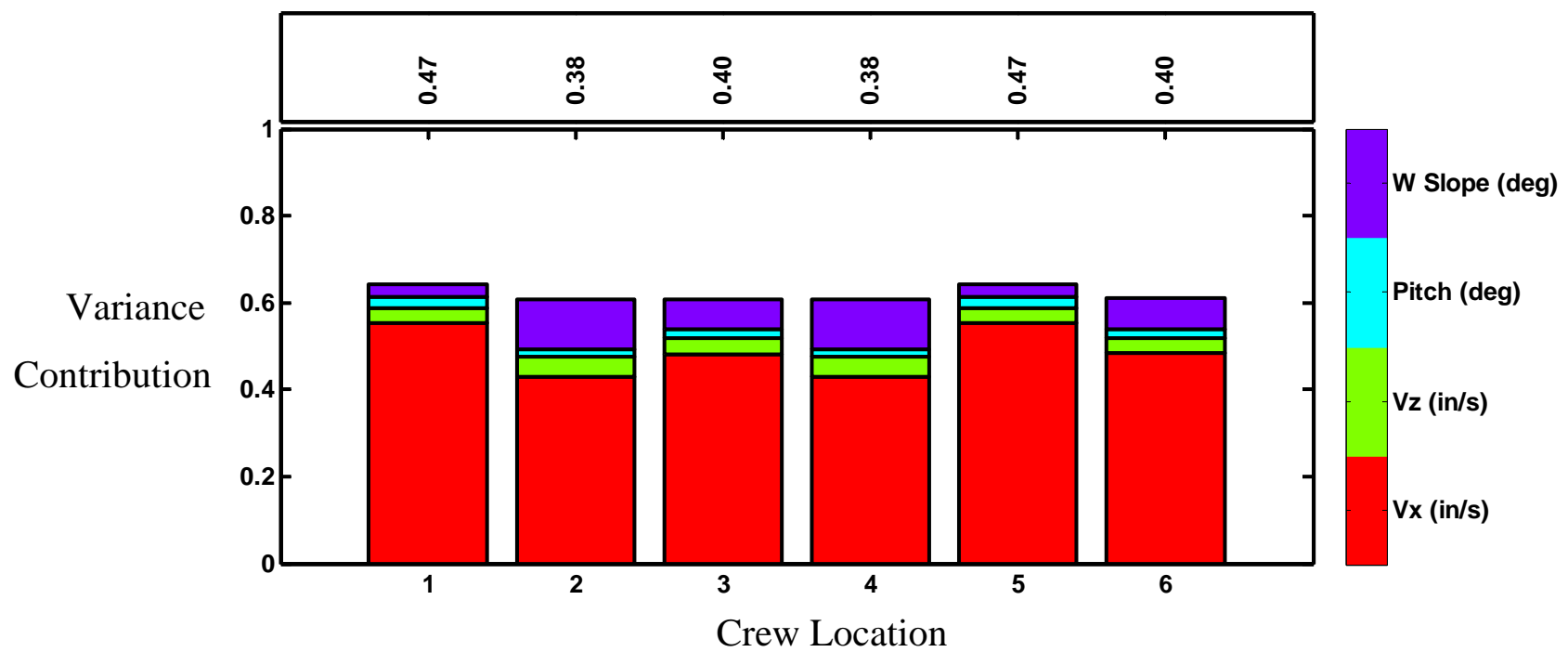

Fig. 9 Variance analysis of Brinkley index using the 4 parameter RS model.

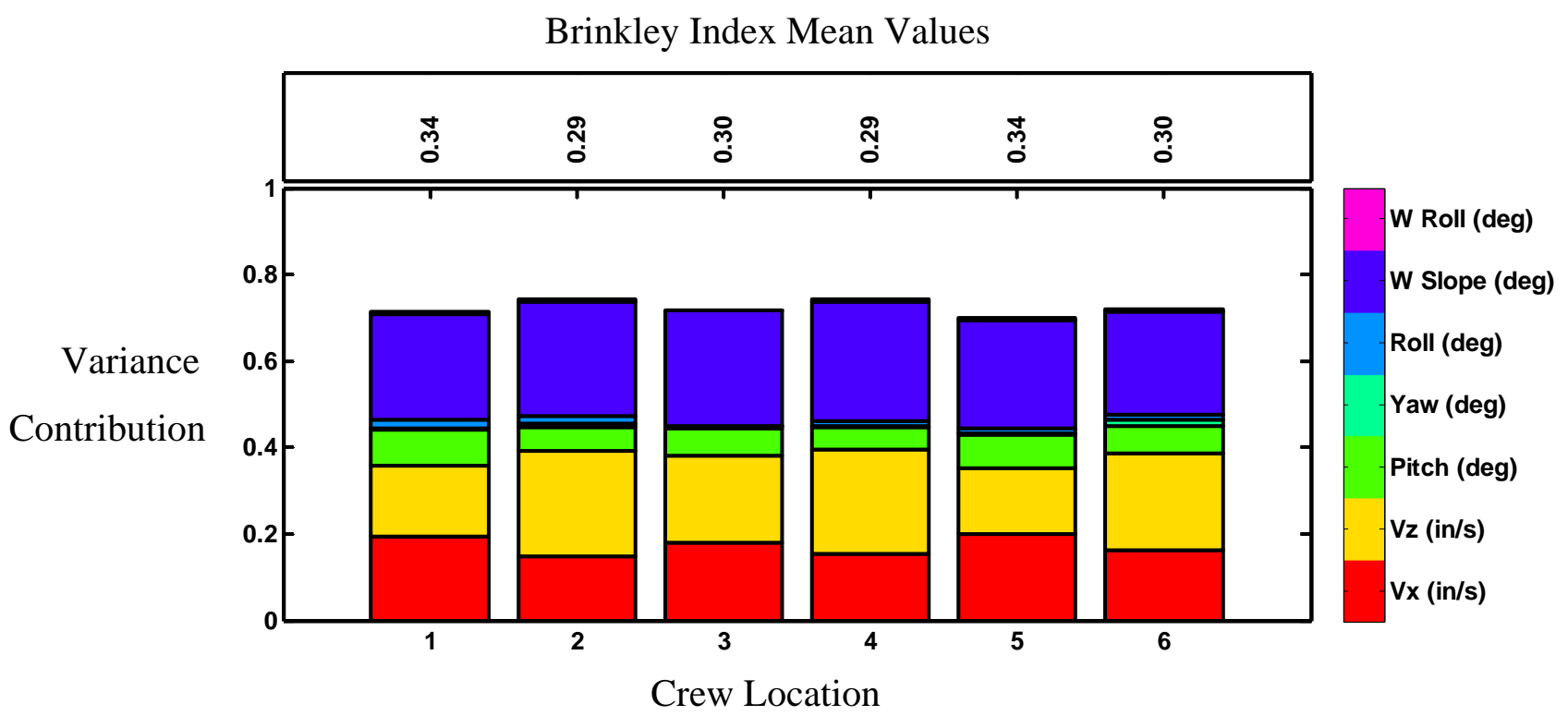

Fig. 10 Variance of Brinkley index using the 7 parameter ERBF model. 


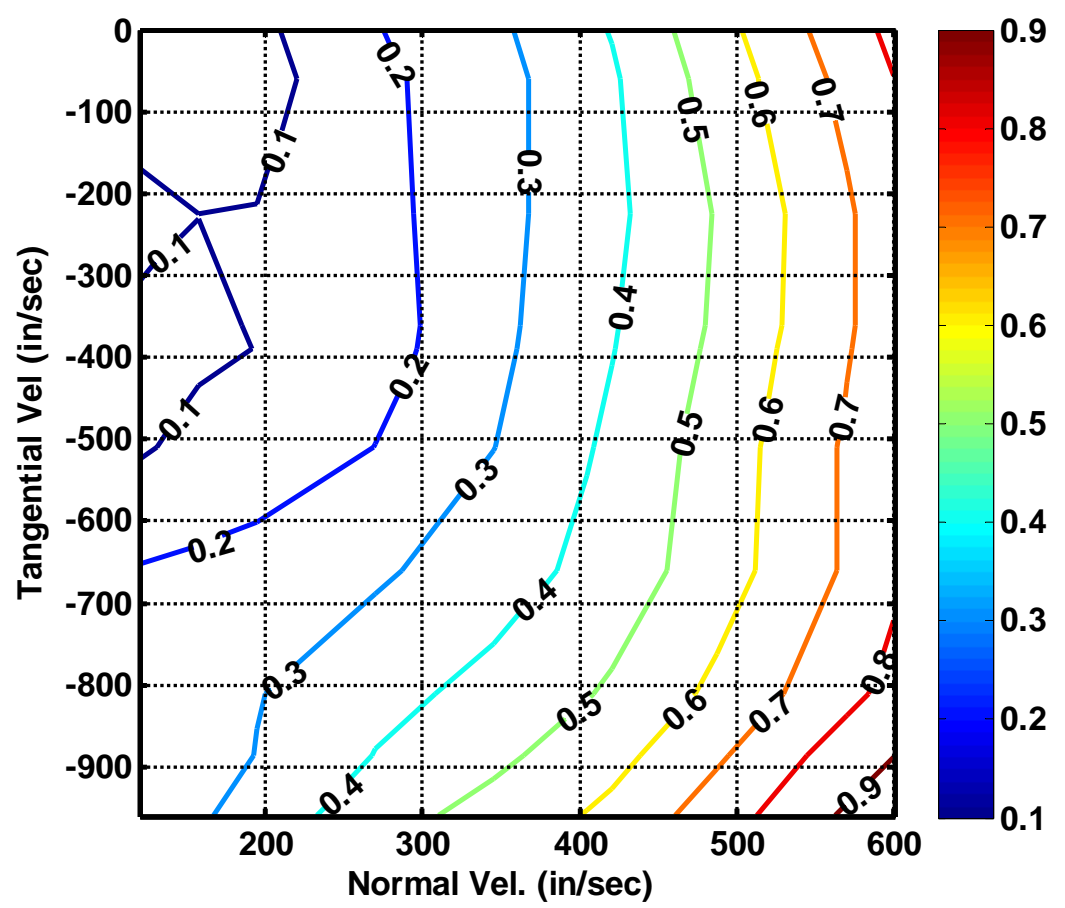

a) 4-Parameter database and 146 LS-DYNA runs

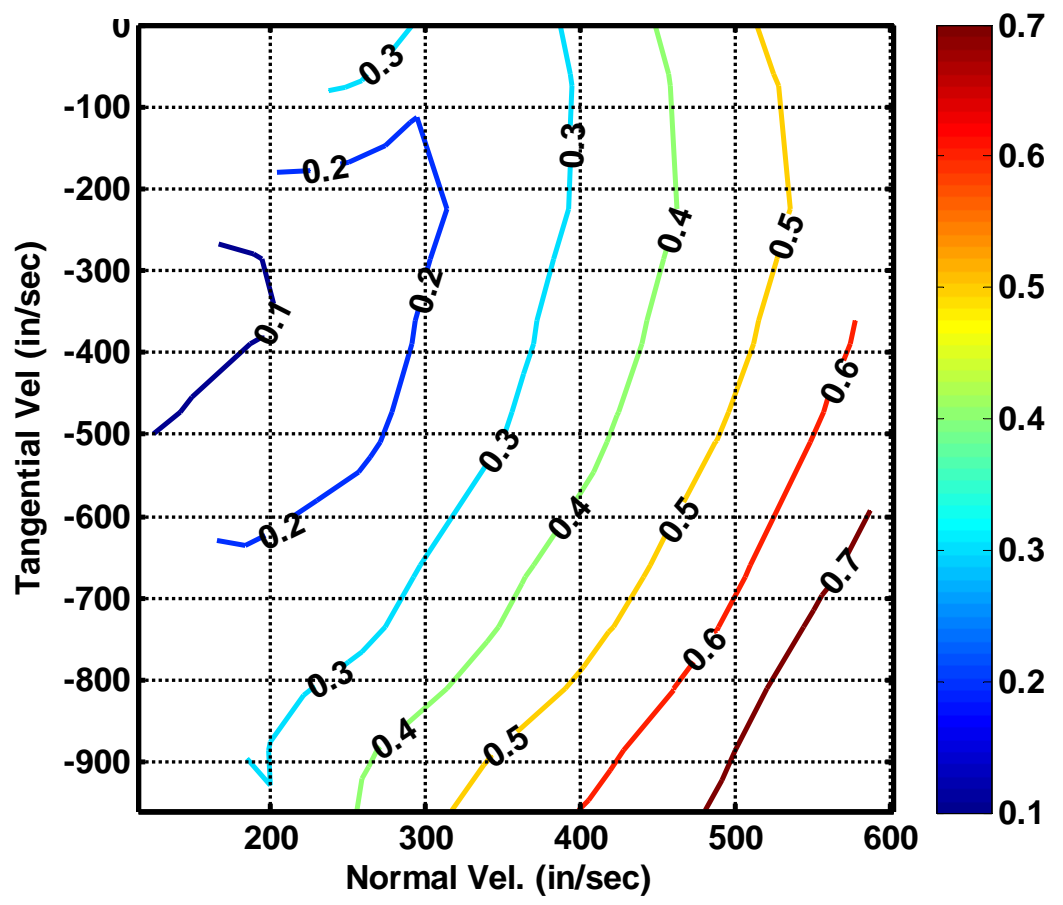

b) 7-Parameter database and 239 LS-DYNA runs

Fig. 11 Comparison of contour plots for the Brinkley index using the 4-parameter and a 7-parameter database. 

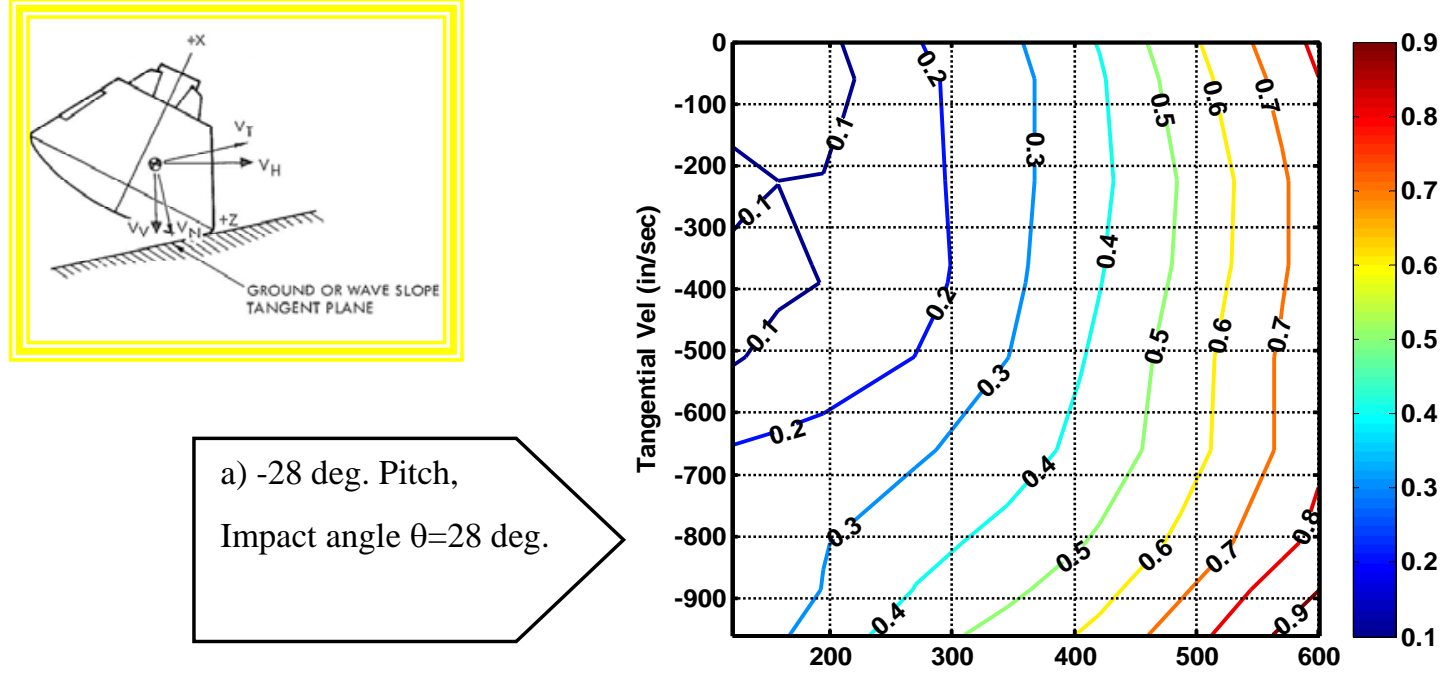

a) -28 deg. Pitch, Impact angle $\theta=28 \mathrm{deg}$.

b) -18 deg. Pitch, Impact angle $\theta=18 \mathrm{deg}$.
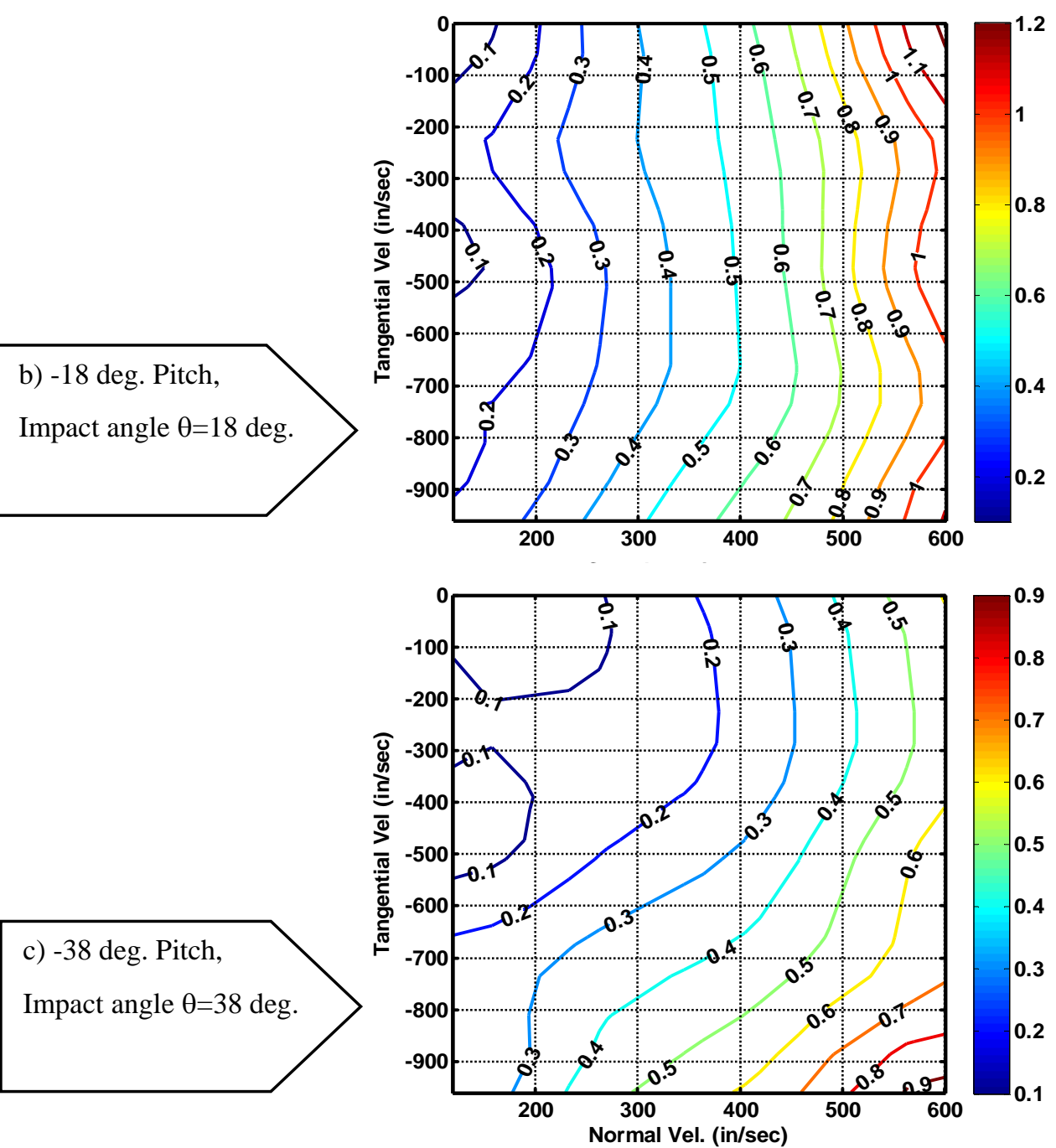

Fig. 12 Contour plots for Brinkley Index as a function of the impact tangential and normal velocity at the crew location 5 . 


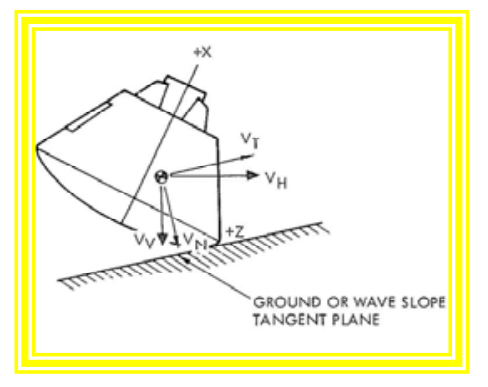

a) 0 deg. Wave Slope, Impact angle $\theta=28 \mathrm{deg}$

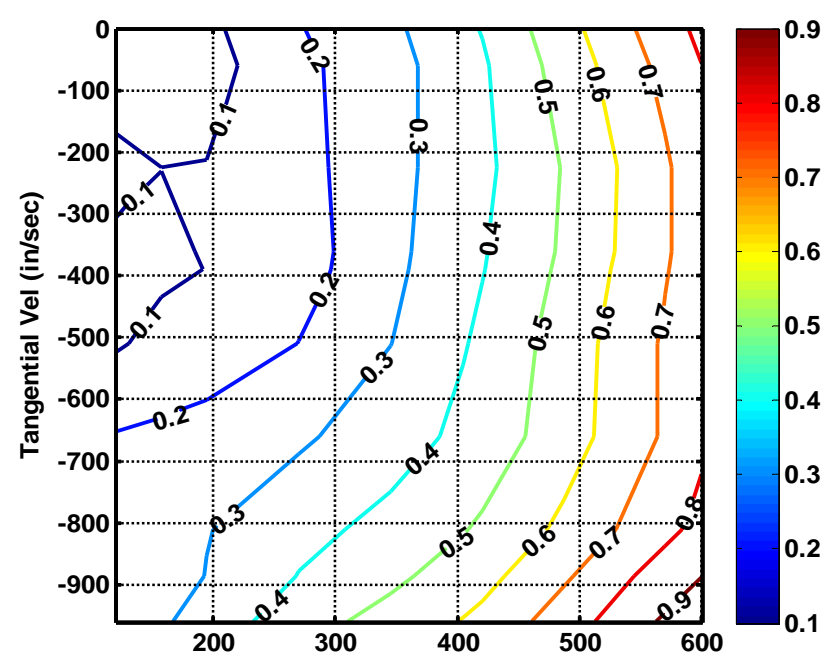

b) 30 deg. Wave Slope, Impact angle $\theta=58 \mathrm{deg}$
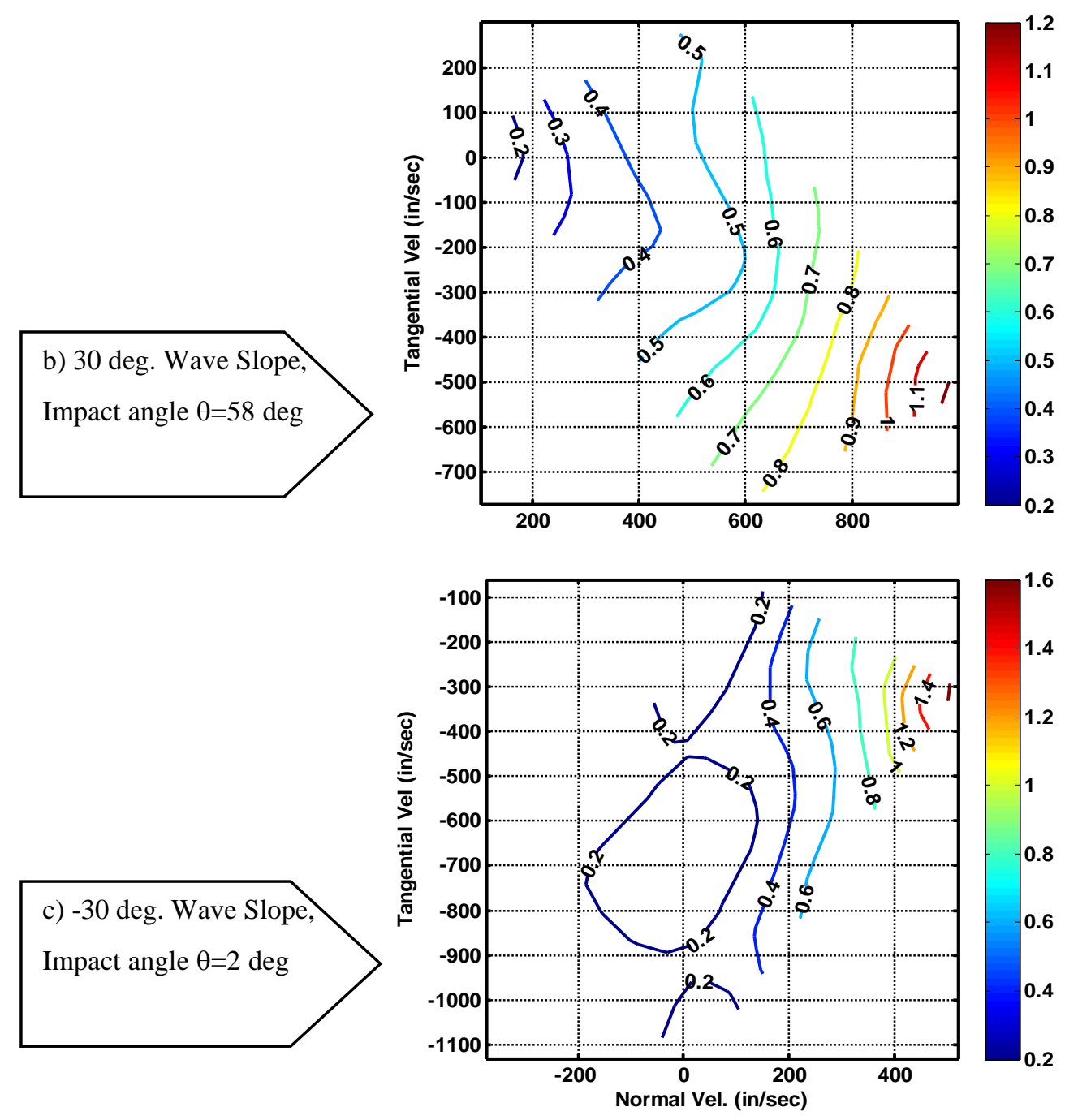

Fig. 13 Contour plots for Brinkley index as a function of the impact tangential and normal velocity at the crew location 5 . 


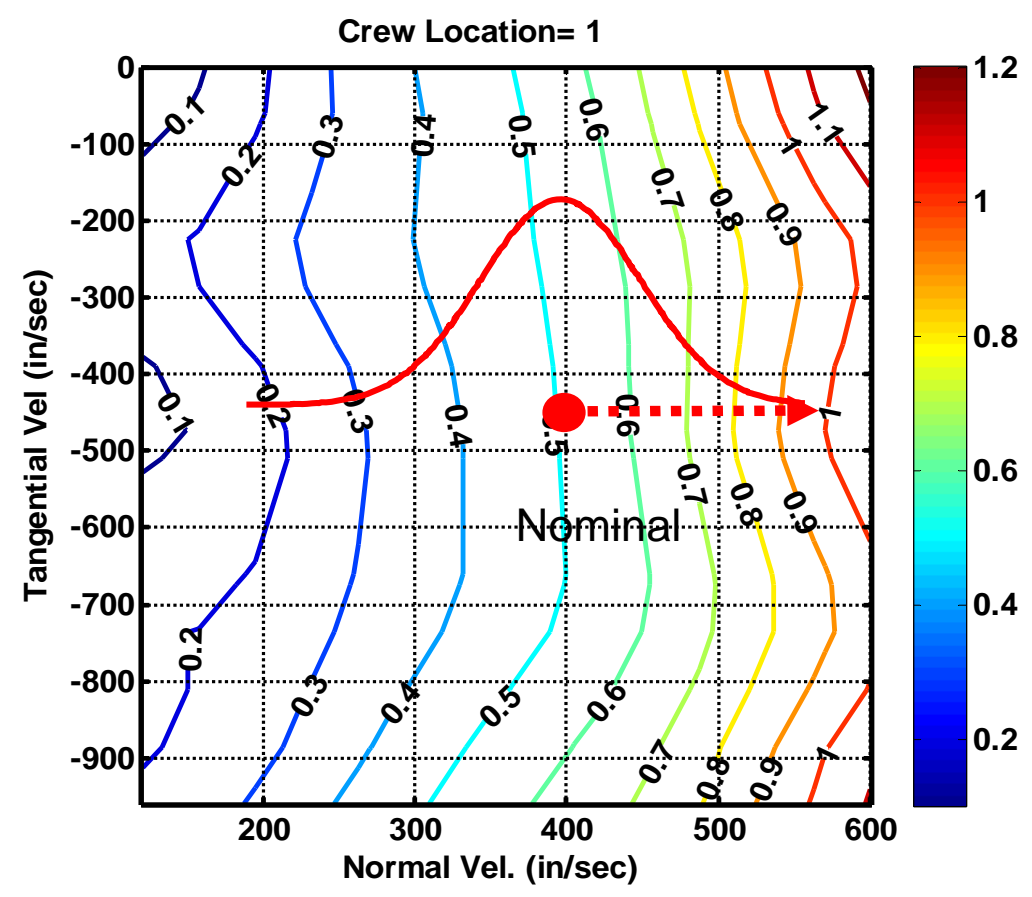

Fig. 14 Illustration of process to estimate most likely critical condition.

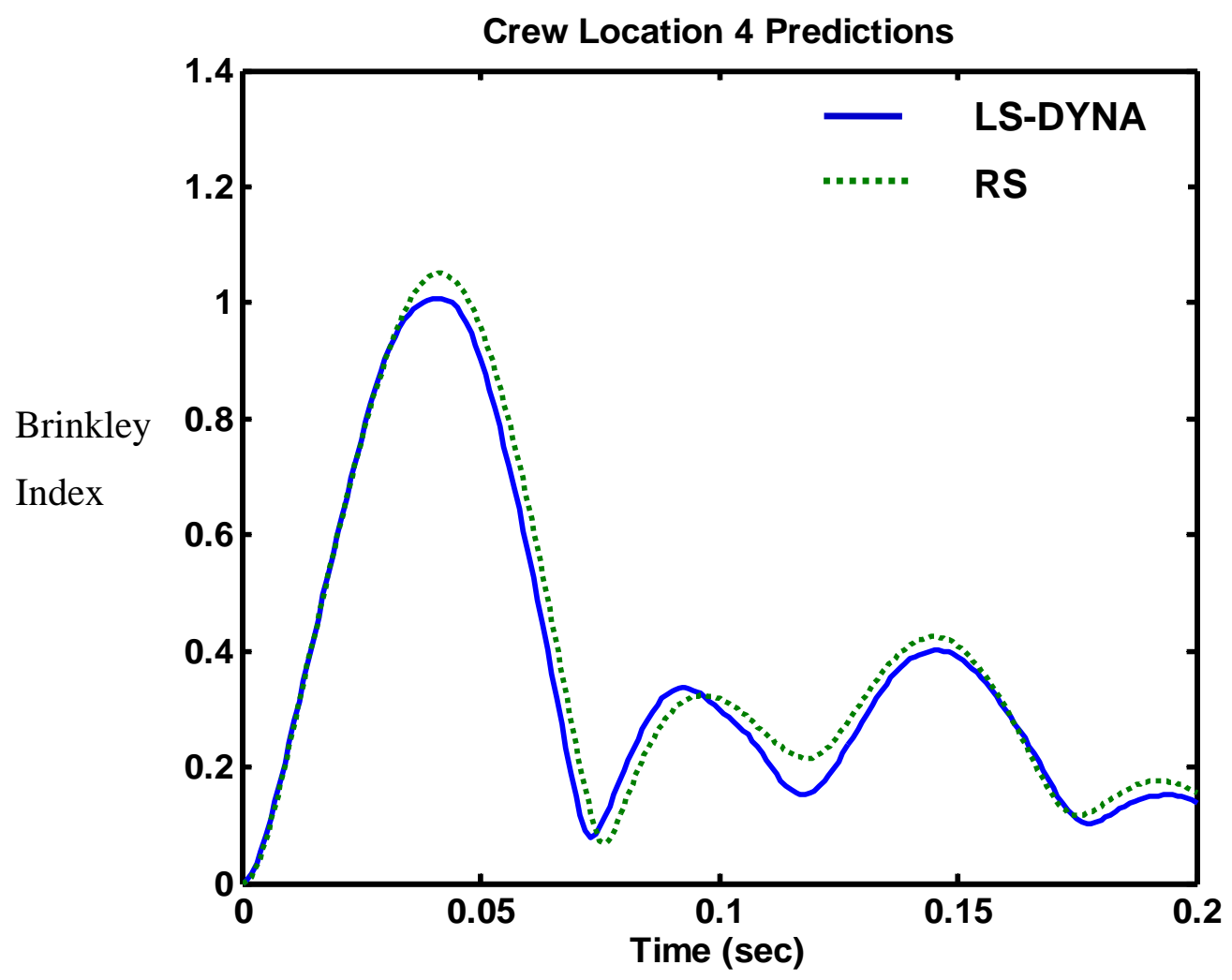

Fig. 15 Verification of predicted critical landing condition using the response surface model. 


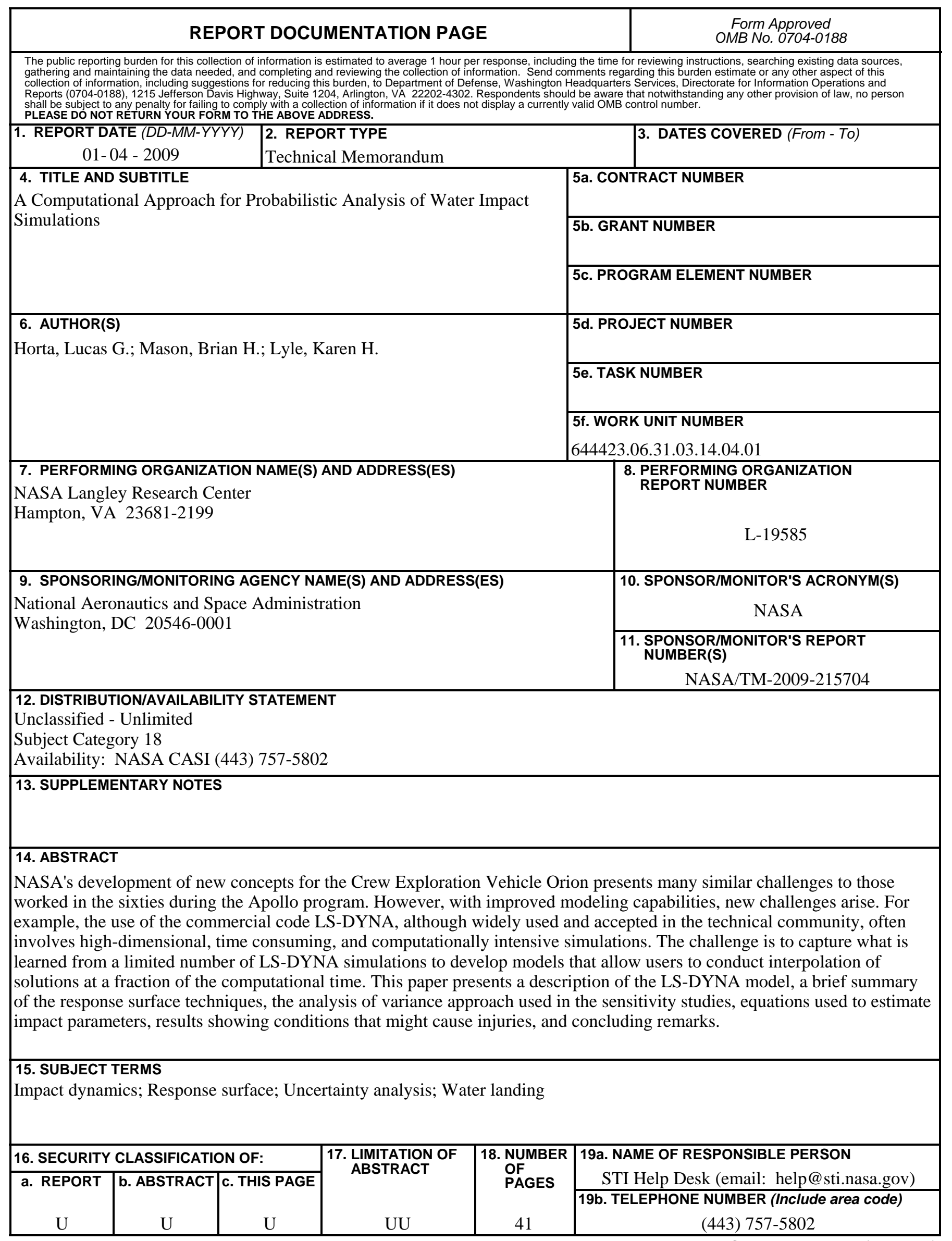

